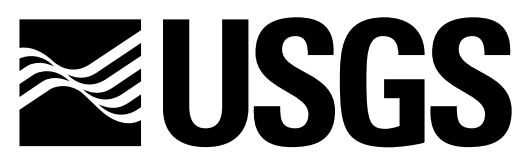

science for a changing world

\title{
Ground-Water Levels and Water-Quality Data from Monitoring Wells in Windham, Maine Water Years 1997-2001
}

Open-File Report 02-145

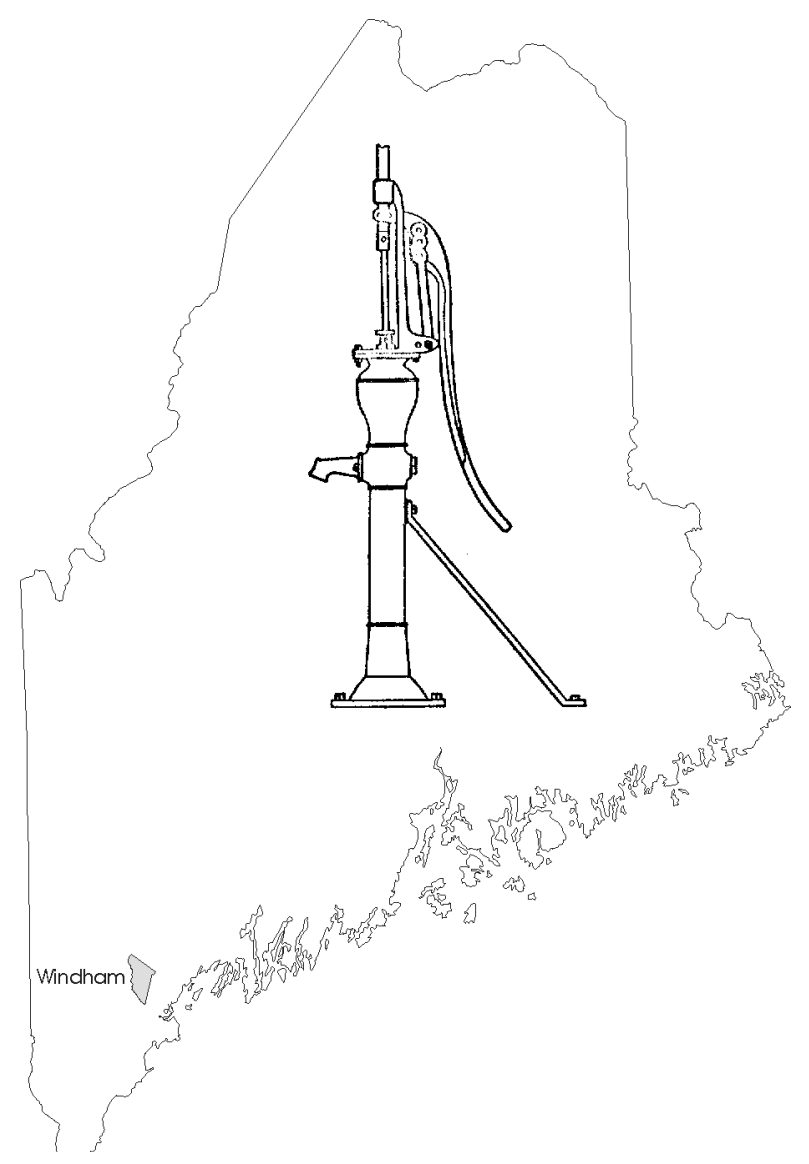

Prepared in cooperation with the Town of Windham, Maine

U.S. Department of the Interior

U.S. Geological Survey 
U.S. Department of the Interior

U.S. Geological Survey

\section{Ground-Water Levels and Water-Quality Data from Monitoring Wells in Windham, Maine Water Years 1997-2001}

By James M. Caldwell

Open-File Report 02-145

Prepared in cooperation with the Town of Windham, Maine 


\section{U.S. DEPARTMENT OF THE INTERIOR \\ GALE A. NORTON, Secretary}

U.S. GEOLOGICAL SURVEY

Charles G. Groat, Director

The use of firm, trade, and brand names in this report is for identification purposes

only and does not constitute endorsement by the U.S. Government.

For additional information write to:

District Chief

U.S. Geological Survey

26 Ganneston Dr.

Augusta, ME 04330

http://me.water.usgs.gov
Copies of this report can be purchased from:

U.S. Geological Survey

Branch of Information Services

Box 25286, Federal Center

Denver, CO 80225 


\section{CONTENTS}

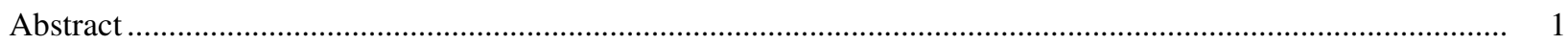

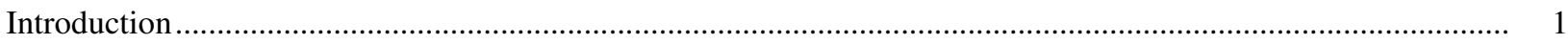

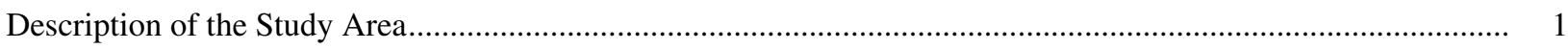

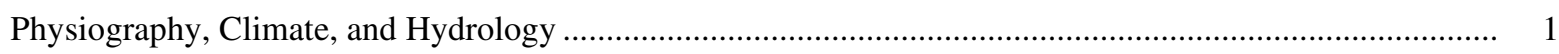

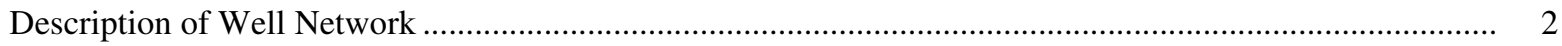

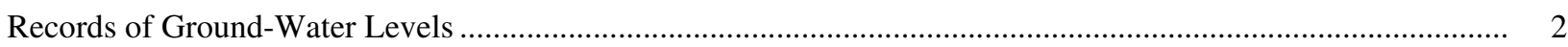

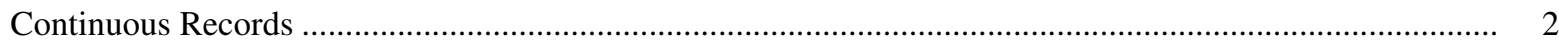

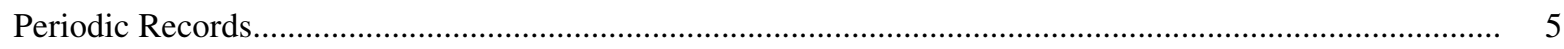

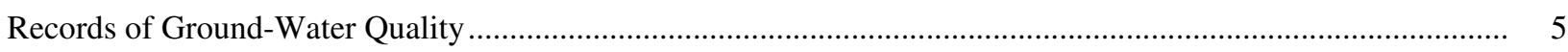

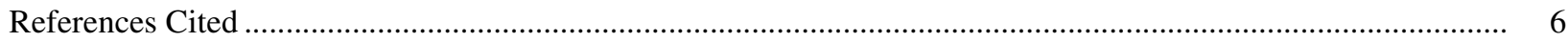

\section{FIGURES}

1. Map showing location of the Windham aquifer study area, North Windham, Maine ................................. 2

2. Map showing monitoring wells in the Windham aquifer, North Windham, Maine..................................

3. Graph showing daily mean water levels in well CW 1983, October 1, 1995 to September 30, 2001......... 6

\section{TABLES}

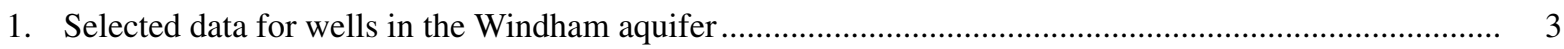

2. Water level data for monitoring well CW 1983, water years 1996-01 .............................................. 8

3. Water-quality data from the Windham aquifer, Windham, Maine, 1998-01 ....................................... 14 
CONVERSION FACTORS AND VERTICAL DATUM

\begin{tabular}{rll}
\hline Multiply & By & To obtain \\
\hline inch (in.) & 2.54 & centimeter \\
foot (ft) & 0.3048 & meter \\
mile (mi) & 1.609 & kilometer \\
square mile $\left(\mathrm{mi}^{2}\right)$ & 2.59 & square kilometer \\
gallons $(\mathrm{gal})$ & 3.785 & liter \\
\hline
\end{tabular}

Temperature in degrees Celsius $\left({ }^{\circ} \mathrm{C}\right)$ may be converted to temperature in degrees Fahrenheit $\left({ }^{\circ} \mathrm{F}\right)$ as follows:

$$
{ }^{\circ} \mathrm{F}=1.8 *\left({ }^{\circ} \mathrm{C}\right)+32
$$

Temperature in degrees Fahrenheit $\left({ }^{\circ} \mathrm{F}\right)$ may be converted to temperature in degrees Celsius $\left({ }^{\circ} \mathrm{C}\right)$ as follows:

$$
{ }^{\circ} \mathrm{C}=\left({ }^{\circ} \mathrm{F}-32\right) / 1.8
$$

Sea level: In this report "sea level" refers to the National Geodetic Vertical Datum of 1929 (NGVD of 1929)—a geodetic datum derived from a general adjustment of the first-order level nets of both the United States and Canada, formerly called Sea Level Datum of 1929.

Altitude: In this report refers to distance above or below sea level.

Specific conductance is given in microsiemens per centimeter at 25 degrees Celsius $\left(\mu \mathrm{S} / \mathrm{cm}\right.$ at $\left.25{ }^{\circ} \mathrm{C}\right)$.

Concentrations of chemical constituents in water are given in milligrams per liter (mg/L) or micrograms per liter ( $\mu \mathrm{g} / \mathrm{L})$. 


\title{
Ground-Water Levels and Water-Quality Data from Monitoring Wells in Windham, Maine, Water Years 1997-2001
}

\author{
by James M. Caldwell
}

\begin{abstract}
Ongoing data collection in an established well network in Windham, Maine, serves as an indicator of the hydrologic and water-quality conditions in the aquifer. This report presents data collected from 1997 through 2001, including ground-water levels, measurements of waterquality field parameters, and concentrations of nutrients and arsenic.
\end{abstract}

\section{INTRODUCTION}

The Town of Windham is one of Maine's fastest growing communities and one of the largest communities in the State without sewerage. Continued growth poses potential risks to the quality of ground water. A common indicator of ground-water contamination is elevated concentrations of nutrients. The nutrients are typically derived from sewage, fertilizer, and animal or landfill waste. One of the primary recommendations of an earlier ground-water evaluation (Gerber, Inc., 1997) was to monitor ground-water quality.

The USGS and the Town of Windham have had an ongoing cooperative relationship for many years. A network of monitoring wells was established in 1995, as part of an investigation of the sand and gravel aquifer in North Windham (Nichols and Silverman, 1998). A study on the occurrence of methyl tert-butyl ether (MTBE) was done in 1998 with the USGS, the Maine Department of Environmental Protection, and the Town of Windham (Nielsen and Peckenham, 2000). Most recently, in 2000, the USGS and the Town of Windham began a 3-year low intensity data-collection program to monitor hydrologic and water-quality conditions in the aquifer. Monitoring the quality of water in the established well network will help determine if there is degradation of ground-water quality and will help Windham's Planning Committee make informed decisions regarding ground-water quality.

This report presents hourly ground-water level data from a representative well in the study area. It also contains water-quality data collected from 1997 to 2001 in monitoring wells in the network. Specifically, it includes nutrient data from samples collected at 31 observation wells in 1998, at 20 observation wells in September 2000, and at 21 observation wells in August 2001. Samples collected in September 2000 also were analyzed for arsenic, to augment statewide studies underway by the Maine State Department of Health.

\section{DESCRIPTION OF THE STUDY AREA}

The Town of Windham lies $12 \mathrm{mi}$ northwest of Portland in Cumberland County, Maine. The study area (fig. 1) is in North Windham, on the North Windham 7.5-minute topographic quadrangle. The population of Windham was 6,593 in $1970,13,020$ in 1990, and 15,600 in 2000 (Maine Register, 2000).

Land use consists primarily of commercial and residential areas. Most residential and commercial growth in Windham has been in North Windham. Restaurants, retail development, and light industrial businesses are mainly along U.S. Route 302, which runs north/south through North Windham.

\section{Physiography, Climate, and Hydrology}

The study area is bordered by hills to the north and northeast, Sebago Lake to the west, and Little Sebago Lake on the east. The terrain consists primarily of low relief and rolling hills to the south. 


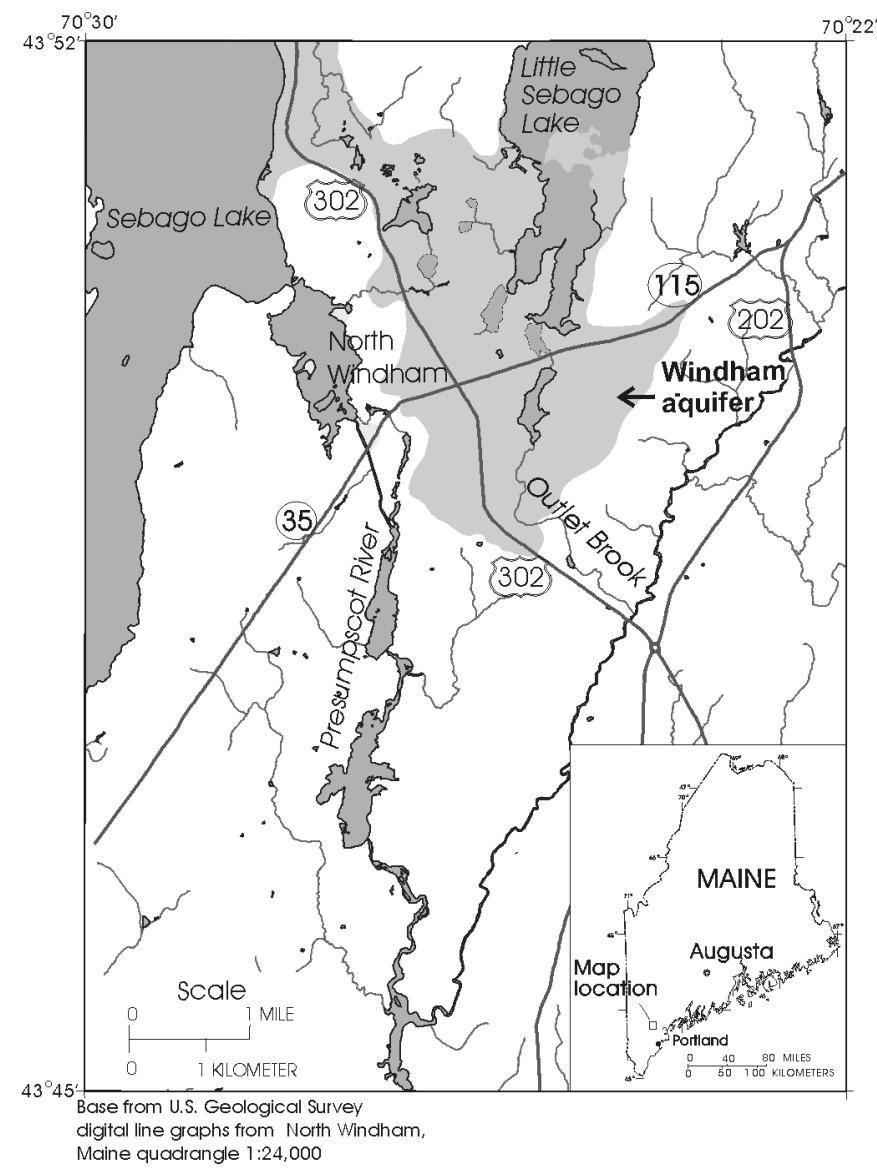

Figure 1. Location of the Windham aquifer study area, North Windham, Maine.

The climate in the Windham area is typified by mild summers and cold winters. Records from a National Weather Service (NWS) station in Lewiston, Maine (14 mi to the northeast), with 114 years of record, indicate an average annual temperature of $46^{\circ} \mathrm{F}$, with mean monthly temperatures ranging from $20^{\circ} \mathrm{F}$ in January to $71{ }^{\circ} \mathrm{F}$ in July (U.S. Department of Commerce, National Oceanic and Atmospheric Administration, 1999). The average annual precipitation at the Lewiston station, with 125 years of record, is $45.8 \mathrm{in}$. and is fairly evenly distributed throughout the year (U.S. Department of Commerce, National Oceanic and Atmospheric Administration, 1992).

The Windham aquifer is termed a "significant sand and gravel aquifer," with yields greater than 10 to $50 \mathrm{gal} / \mathrm{min}$ to a properly constructed well (Neil, 1999). The aquifer is composed primarily of glaciomarine deltic sands and gravels. A buried esker with high hydraulic conductivity lies in the northern part of the aquifer (Gerber, Inc., 1997). Detailed descriptions of the hydrology of the aquifer can be found in Nielsen and Peckenham (2000) and Gerber, Inc. (1997).

\section{Description of Well Network}

The primary identification number for each well in the network is the 15-digit USGS station identification number (table 1). The secondary identification number is the local well number, an alphanumeric number, composed of an abbreviation of the county name and sequential number (fig. 2).

Well depths range from 19 to $132 \mathrm{ft}$. The screened intervals are mostly 5 or $10 \mathrm{ft}$, with a few wells having longer screens (20 or $30 \mathrm{ft}$ ). Depths of the screened intervals of the wells range from 8 to $130 \mathrm{ft}$.

\section{RECORDS OF GROUND-WATER LEVELS}

\section{Continuous Records}

One observation well in the aquifer, CW 1983, is equipped with a downhole recorder that records hourly ground-water levels. Ground-water levels for water years 1996-2001 are shown in table 2. Beginning with water year 2000, ground-water level data also are published in the annual data report series (Stewart and others, 2000). Water levels observed during the period of record with the dates of ground-water sample collected are shown in figure 3 . 
Table 1. Selected data for wells in the Windham aquifer

[Water-level data modified (values rounded) from Nichols and Silverman (1998). Aquifer codes: IC, ice-contact deposits; GL, glacial sediments, undifferentiated; TL, till; $\mathrm{M}$, marine; $\mathrm{D}$, deltaic deposits. --, no data available]

\begin{tabular}{|c|c|c|c|c|c|c|c|}
\hline $\begin{array}{c}\text { U.S. Geological Survey } \\
\text { station identification } \\
\text { number }\end{array}$ & $\begin{array}{l}\text { Local well } \\
\text { number }\end{array}$ & $\begin{array}{l}\text { Well } \\
\text { depth, } \\
\text { in feet }\end{array}$ & $\begin{array}{l}\text { Land surface } \\
\text { elevation, } \\
\text { in feet above } \\
\text { sea level }\end{array}$ & $\begin{array}{l}\text { Aquifer } \\
\text { code }\end{array}$ & $\begin{array}{c}\text { Screened } \\
\text { interval, } \\
\text { in feet below } \\
\text { land surface }\end{array}$ & $\begin{array}{l}\text { Screen } \\
\text { length, } \\
\text { in feet }\end{array}$ & $\begin{array}{l}\text { Water leve } \\
\text { range, } \\
\text { in feet } \\
\text { below land } \\
\text { surface }\end{array}$ \\
\hline 435002070255701 & CW 1971 & 22 & 299.8 & IC & $17-22$ & 5 & $12-16$ \\
\hline 434934070244101 & CW 1975 & 47.5 & 273.9 & M & $32.5-37.5$ & 5 & $21-26$ \\
\hline 435008070253901 & CW 1979 & 109 & 309.1 & $\mathrm{D}$ & $104-109$ & 5 & $37-39$ \\
\hline 434919070262601 & CW 1980 & 36 & 307.1 & IC & $31-36$ & 5 & $14-18$ \\
\hline 435018070250201 & CW 1981 & 57.7 & 312.9 & IC & $44-49$ & 5 & $22-28$ \\
\hline 434958070261601 & CW 1982 & 55 & 297.5 & GL & $40-45$ & 5 & $15-18$ \\
\hline 435039070261101 & CW 1983 & 37.6 & 308.0 & GL & $32-37$ & 5 & $14-18$ \\
\hline 435056070263701 & CW 1984 & 132 & 295.1 & IC & $120-130$ & 10 & $3-7$ \\
\hline 435055070263601 & CW 1985 & 25.7 & 295.1 & IC & $15.5-25.5$ & 10 & $3-7$ \\
\hline 434910070255601 & CW 1987 & 111 & 303.5 & IC & $101-111$ & 10 & $58-60$ \\
\hline 434941070261901 & CW 1988 & 55 & 290.6 & GL & $48-55$ & 7 & $1-4$ \\
\hline 434919070262602 & CW 1989 & 19 & 307.0 & GL & $14-19$ & 5 & $11-18$ \\
\hline 435002070255601 & CW 1990 & 80 & 299.9 & $\mathrm{IC}$ & $60-80$ & 20 & $25-28$ \\
\hline 435008070262901 & CW 1992 & 54 & 305.0 & GL & $37-42$ & 5 & $10-14$ \\
\hline 435008070262902 & CW 1993 & 27 & 305.0 & GL & $22-27$ & 5 & $8-11$ \\
\hline 434945070263401 & CW 1994 & 20 & 276.7 & $\mathrm{TL}$ & $10-15$ & 5 & $0-4$ \\
\hline 435012070265101 & CW 1995 & 52 & 307.5 & $\mathrm{TL}$ & $22-32$ & 10 & $11-15$ \\
\hline 435027070264801 & CW 1998 & 35 & 312.0 & GL & $30-35$ & 5 & $6-20$ \\
\hline 435026070264101 & CW 1999 & 80 & 310.8 & GL & $78-80$ & 8 & $18-23$ \\
\hline 435041070262301 & CW 2000 & 70 & 319.7 & GL & $40-50$ & 10 & $26-28$ \\
\hline 435131070261401 & CW 2001 & 127 & 309.6 & GL & $97-127$ & 30 & $13-16$ \\
\hline 434946070252301 & CW 2003 & 67 & 316.2 & GL & $57-62$ & 5 & $44-46$ \\
\hline 434927070255101 & CW 2004 & 58 & 315.0 & GL & $38-58$ & 20 & $49-51$ \\
\hline 435008070253902 & CW 2005 & 48 & 309.0 & GL & $38-48$ & 10 & $34-36$ \\
\hline 435040070264501 & CW 2007 & 24 & 301.4 & GL & $14-24$ & 10 & $8-10$ \\
\hline 435131070261402 & CW 2008 & 27 & 309.5 & GL & $17-27$ & 10 & $13-15$ \\
\hline 435026070264102 & CW 2009 & 22 & 310.8 & GL & $12-22$ & 10 & $11-14$ \\
\hline 435018070263201 & CW 2010 & 32 & 310.7 & GL & $22-32$ & 10 & $14-16$ \\
\hline 435014070264901 & CW 2011 & 27 & 310.7 & GL & $17-27$ & 10 & $12-14$ \\
\hline 435004070262101 & CW 2012 & 27 & 307.6 & GL & $17-27$ & 10 & $13-16$ \\
\hline 435136070271201 & CW 2019 & -- & 312.8 & -- & $8.1-13.1$ & 5 & $3-9$ \\
\hline
\end{tabular}




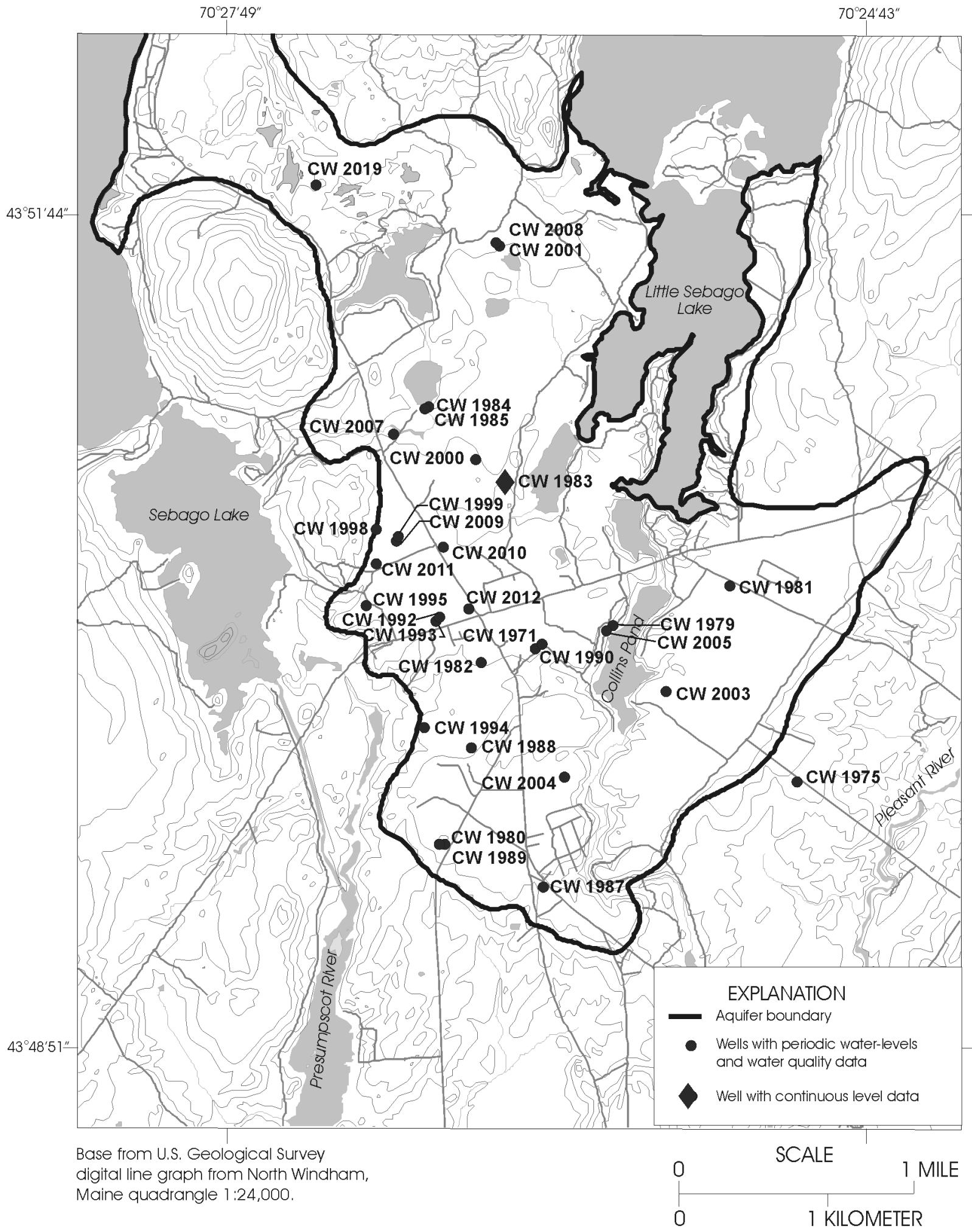

Figure 2. Monitoring wells in the Windham aquifer, Windham, Maine. [Aquifer boundary from Neil, 1998.] 
Water levels are reported in feet below land-surface datum. Land-surface datum is a datum plane that is approximately at land surface at each well and does not vary. The highest and lowest water levels for each month are shown on a line below the daily-mean table. Missing records are indicated by dashes in place of the water level.

\section{Periodic Records}

Water levels also were measured with a tape while collecting water-quality samples at all network wells; these levels are shown with the water-quality data (table 3). The water-level measurements in this report are given in feet below land-surface datum.

Both flood and drought conditions occurred in the study area during the sampling period. Up to 19.19 in. of rain were recorded in southern Maine from October 20-22, 1996 (Hodgkins and Stewart, 1997). The maximum 24-hour rainfall total (13.32 in.) at the Portland International Jetport during this period exceeded the previous record by 5.49 in. (Records have been kept since 1871.) The recharge response from this event can be seen in CW 1983 (table 2 and fig. 3). In addition, ground-water levels were above normal in the spring and summer of 1998 and were below normal during 1999, with lows in early September just before Hurricane Floyd brought substantial recharge. Ground-water levels were in the normal range during most of 2000. Levels were in the normal and below-normal ranges for most of water year 2001 (Nielsen and others, 1997-99; Stewart and others, 2000).

\section{RECORDS OF GROUND-WATER QUALITY}

In 1998, the USGS, in cooperation with the Maine Department of Environmental Protection and the Town of Windham, began a study of the occurrence of MTBE (Nielsen and Peckenham, 2000). Thirty-one monitoring wells in the Windham aquifer were sampled from July 1998 to May 1999. Samples also were analyzed for major nutrients, and those nutrient data are presented in this report (table 3).

In 2000, the USGS, in cooperation with the Town of Windham, began a 3-year low intensity datacollection program to serve as an indicator of the hydrologic and water-quality conditions in the aquifer. Using the existing network of observation wells, samples were collected in September 2000 at 20 wells and in August 2001 at 21 wells.

A submersible Fultz 300R pump with Teflonlined tubing was used to collect all samples. The Teflon outflow tube was connected to a Hydrolab multi-parameter monitor with flow-through cell for measuring field values (the use of trade names in USGS reports is for identification purposes only and is not intended as an endorsement). Once the field values stabilized (did not change more than 5 percent for three consecutive 3- to 5-minute measurements) and drawdown was kept to within 20 percent of the standing head, the flow rate was measured and the sample collected. Flow rates generally ranged from 0.026 to $0.12 \mathrm{gal} / \mathrm{min}(100 \mathrm{~mL} / \mathrm{min}$ to $400 \mathrm{~mL} / \mathrm{min})$. Samples were filtered through a 0.45 -micron filter, kept on ice and shipped within 24 hours to the USGS laboratory in Arvada, Colorado, for analysis. After sampling, all the equipment was cleaned with a dilute Liquinox solution and rinsed with a copious amount of distilled water. During the 1998 and 1999 MTBE study, all equipment also was rinsed well with methanol and rinsed again with distilled water because samples were being analyzed for VOCs. The pump and cleaned tubing were packed in plastic sheeting for transport between sites.

USGS National Water Quality Assessment protocols for sampling ground-water wells (Koterba and others, 1995) were followed in sample handling, quality assurance/quality control, sampling equipment, and cleaning methods. The USGS protocols were modified to follow the U.S. Environmental Protection Agency (USEPA) low-flow (minimal drawdown) sampling procedures (Puls and Barcelona, 1995). Three wells did not yield enough water to meet the minimum drawdown requirements. These wells (CW 1980, CW 1981, CW 1999) were pumped dry and sampled the following day.

Specific conductance, dissolved oxygen, $\mathrm{pH}$, temperature and (in 1998) alkalinity were measured in the field. All other constituents were analyzed in the USGS laboratory using methods described by Fishman (1993). 


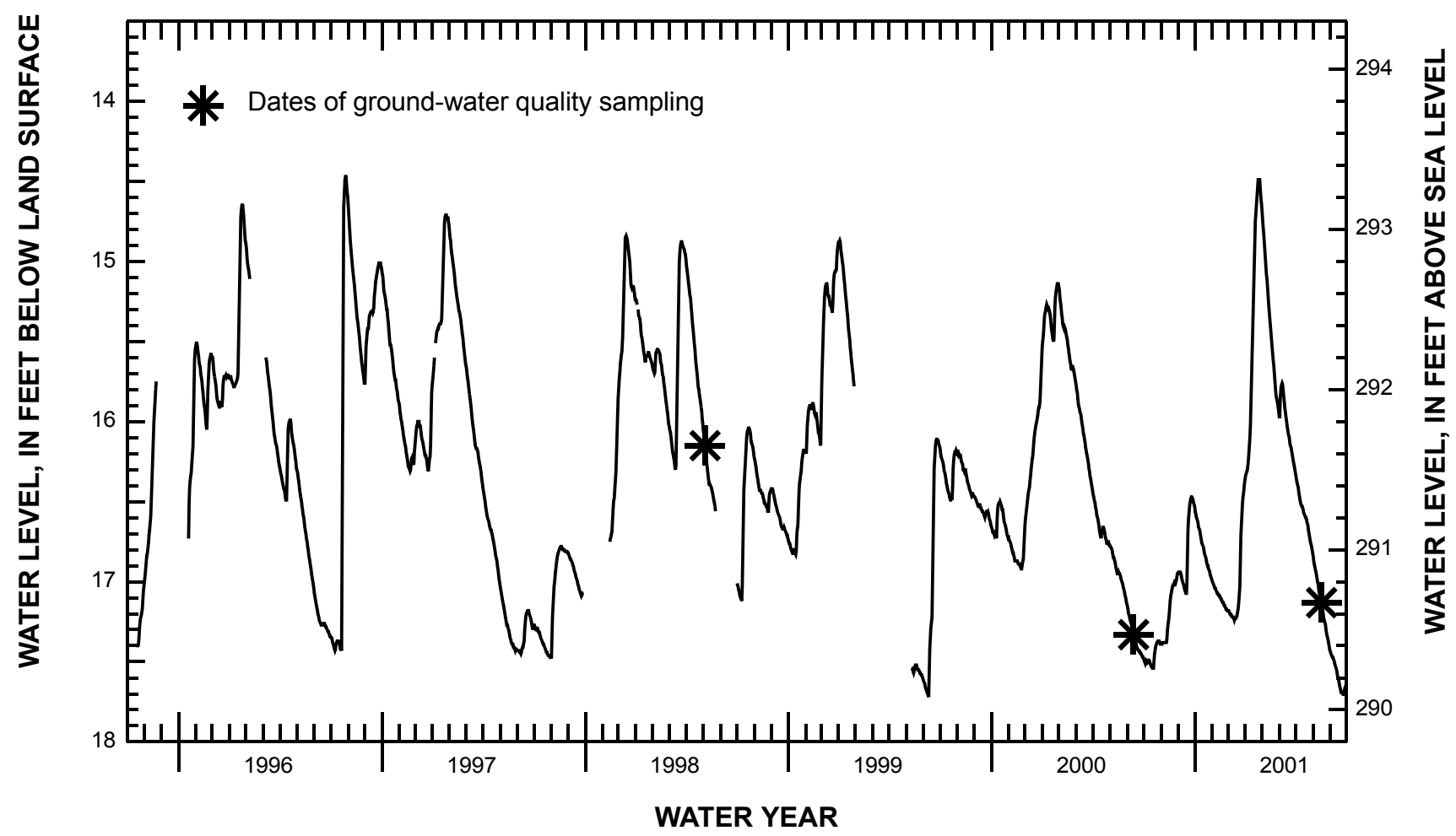

Figure 3. Daily mean water levels in monitoring well CW 1983, October 1, 1995 to September 30, 2001.

To ensure collection of representative samples from the aquifer, samples were pumped from the screened interval of the observation wells. In addition, about 12 percent of the water samples were qualitycontrol samples used to assess the accuracy and precision of the water-quality data collected. The following quality-control samples were collected:

(1) Field blank-Distilled water was passed through the pump tubing that the regular samples contact and filtered through a 0.45 -micron filter. These samples were preserved and analyzed along with regular water samples. Results are used to assess equipment cleaning procedures and identify possible contamination introduced by sample handling and transport.

(2) Duplicate-A second sample was collected at a site; it was filtered, preserved, and analyzed along with the regular samples. Results are used to estimate the combined effects of processing and laboratory precision.

Nutrients were not detected in any of the field blank samples. No significant variability was observed for any constituent between the original and duplicate samples.

\section{REFERENCES CITED}

Fishman, M.J., 1993, ed., Methods of analysis by the U.S. Geological Survey National Water Quality Laboratory-Determination of inorganic and organic constituents in water and fluvial sediments: U.S.Geological Survey Open-File Report 93-125, 217 p.

Gerber, Robert G. Inc., 1997, Windham ground water resource evaluation, Phase 2: Freeport, Maine, Robert G. Gerber, Inc., report dated May 15, 1997, 26 p., plus tables, figures, appendixes, sheets.

Hodgkins, G.A., and Stewart, G.J., Flood of October 1996 in Southern Maine: U.S. Geological Survey Water-Rescources Investigations Report 97-4189, 28 p.

Koterba, M.T., Wilde, F.D., and Lapham, W.W., 1995, Ground-water data-collection protocols and procedures for the National Water-Quality Assessment ProgramCollection and documentation of water-quality samples and related data: U.S. Geological Survey Open-File Report 95-399, 113 p.

Maine Register, 2000, 2001 State Yearbook and Legislative Manual: Standish, Maine, Tower Publishing Co., no. $131,1161 \mathrm{p}$.

Neil, C.D., 1999, Significant sand and gravel aquifers of the Gray quadrangle, Maine: Maine Geological Survey, Open-File Map 99-24. 
Nichols, W.J., and Silverman, P.N., 1998 Hydrologic data for the Presumpscot River basin, Cumberland and Oxford Counties, Maine, 1995 to 1996: U.S.Geological Survey Open-File Report 98-265, 53 p.

Nielsen, M.G., and Peckenham, J.M., 2000, Methyl ter-butyl ether (MTBE) in ground water, air, and precipitation at North Windham, Maine: U.S. Geological Survey

Water-Resources Investigations Report 00-4048 28 p.

Puls, R.W., and Barcelona, M.J., 1995, Low-flow (minimal drawdown) ground-water sampling procedures: U.S. Environmental Protection Agency Research Brief EPA/540/S-95/504.

Nielsen, J.P., Lippert, R.G., Caldwell, J.M., 1997, Water resources data, Maine, water year 1997: U.S. Geological Survey Water-Data Report ME-97-1, 161 p.

1998, Water resources data, Maine, water year 1998: U.S. Geological Survey Water-Data Report ME-98-1, $177 \mathrm{p}$.

Nielsen, J.P., Stewart, G.J., Caldwell, J.M., 1999, Water resources data, Maine, water year 1999: U.S. Geological Survey Water-Data Report ME-99-1, 194 p.

Stewart, G.J., Nielsen, J.P., Caldwell, J.M., Cloutier, A.R., 2000, Water resources data, Maine, water year 2000: U.S. Geological Survey Water-Data Report ME--00-1, $233 \mathrm{p}$.

U.S. Department of Commerce, National Oceanic and Atmospheric Administration, 1992, Climatological data annual summary, New England: v. 104, no. 13. 1999, Climatological data annual summary, New England: v. 111, no. 13. 
Table 2. Water-level data for monitoring well CW 1983, water years 1996-2001

[USGS identifier - 435039070261101; ---, no data; m, manual measurement]

WATER YEAR 1996 (OCTOBER 1995 TO SEPTEMBER 1996)

DEPTH BELOW LAND SURFACE (WATER LEVEL) (FEET), DAILY MEAN VALUES

\begin{tabular}{|c|c|c|c|c|c|c|c|c|c|c|c|c|}
\hline DAY & $\mathrm{OCT}$ & NOV & $\mathrm{DEC}$ & JAN & $\mathrm{FEB}$ & MAR & APR & MAY & JUN & JUL & AUG & SEP \\
\hline 1 & --- & 16.99 & --- & --- & 15.53 & 15.60 & 15.73 & 14.89 & --- & 16.29 & 16.31 & 17.07 \\
\hline 2 & --- & 16.96 & --- & --- & 15.51 & 15.60 & 15.73 & 14.92 & --- & 16.31 & 16.32 & 17.09 \\
\hline 3 & --- & 16.92 & --- & --- & 15.51 & 15.62 & 15.72 & 14.97 & --- & 16.33 & 16.35 & 17.11 \\
\hline 4 & --- & 16.88 & --- & --- & 15.53 & 15.67 & 15.72 & 15.01 & --- & 16.34 & 16.37 & 17.13 \\
\hline 5 & --- & 16.84 & --- & --- & 15.56 & 15.70 & 15.74 & 15.03 & --- & 16.36 & 16.40 & 17.15 \\
\hline 6 & --- & 16.82 & --- & --- & 15.59 & 15.72 & 15.75 & 15.05 & 15.60 & 16.38 & 16.43 & 17.17 \\
\hline 7 & --- & 16.79 & --- & --- & 15.63 & 15.75 & 15.76 & 15.08 & 15.62 & 16.40 & 16.46 & 17.19 \\
\hline 8 & --- & 16.76 & --- & --- & 15.65 & 15.77 & 15.77 & 15.11 & 15.65 & 16.42 & 16.48 & 17.21 \\
\hline 9 & --- & 16.71 & --- & --- & 15.66 & 15.81 & 15.78 & --- & 15.69 & 16.43 & 16.50 & 17.22 \\
\hline 10 & --- & 16.68 & --- & --- & 15.70 & 15.85 & 15.79 & --- & 15.71 & 16.44 & 16.53 & 17.24 \\
\hline 11 & --- & 16.64 & --- & --- & 15.72 & 15.87 & 15.77 & --- & 15.74 & 16.47 & 16.55 & 17.25 \\
\hline 12 & --- & 16.59 & --- & --- & 15.75 & 15.88 & 15.77 & --- & 15.77 & 16.49 & 16.58 & 17.26 \\
\hline 13 & --- & 16.53 & --- & --- & 15.79 & 15.90 & 15.76 & --- & 15.80 & 16.49 & 16.60 & 17.27 \\
\hline 14 & --- & 16.46 & --- & --- & 15.82 & 15.91 & 15.75 & --- & 15.83 & 16.23 & 16.63 & 17.27 \\
\hline 15 & --- & 16.36 & --- & --- & 15.86 & 15.91 & 15.74 & --- & 15.87 & 16.12 & 16.66 & 17.27 \\
\hline 16 & --- & 16.21 & --- & --- & 15.89 & 15.88 & 15.73 & --- & 15.91 & 16.05 & 16.68 & 17.27 \\
\hline 17 & --- & 16.09 & --- & --- & 15.91 & 15.88 & 15.70 & --- & 15.94 & 16.02 & 16.70 & 17.26 \\
\hline 18 & 17.39 & 16.00 & --- & --- & 15.95 & 15.90 & 15.37 & --- & 15.97 & 16.00 & 16.73 & 17.26 \\
\hline 19 & 17.40 & 15.92 & --- & 16.73 & 16.00 & 15.91 & 15.11 & --- & 16.01 & 15.99 & 16.75 & 17.26 \\
\hline 20 & 17.40 & 15.86 & --- & 16.51 & 16.03 & 15.86 & 14.93 & --- & 16.05 & 15.99 & 16.78 & 17.27 \\
\hline 21 & 17.39 & 15.80 & --- & 16.41 & 16.05 & 15.77 & 14.80 & --- & 16.08 & 16.01 & 16.80 & 17.28 \\
\hline 22 & 17.35 & 15.75 & --- & 16.37 & 15.93 & 15.74 & 14.72 & --- & 16.10 & 16.06 & 16.83 & 17.28 \\
\hline 23 & 17.29 & --- & --- & 16.34 & 15.80 & 15.72 & 14.68 & --- & 16.12 & 16.09 & 16.85 & 17.29 \\
\hline 24 & 17.25 & --- & --- & 16.32 & 15.71 & 15.72 & 14.65 & --- & 16.14 & 16.11 & 16.87 & 17.30 \\
\hline 25 & 17.22 & --- & --- & 16.26 & 15.64 & 15.72 & 14.64 & --- & 16.15 & 16.13 & 16.90 & 17.31 \\
\hline 26 & 17.21 & --- & --- & 16.20 & 15.61 & 15.71 & 14.68 & --- & 16.17 & 16.15 & 16.92 & 17.31 \\
\hline 27 & 17.19 & --- & --- & 16.16 & 15.60 & 15.72 & 14.71 & --- & 16.20 & 16.17 & 16.95 & 17.32 \\
\hline 28 & 17.17 & --- & --- & 15.91 & 15.58 & 15.71 & 14.77 & --- & 16.22 & 16.20 & 16.97 & 17.33 \\
\hline 29 & 17.11 & --- & --- & 15.72 & 15.58 & 15.71 & 14.82 & --- & 16.25 & 16.23 & 16.99 & 17.34 \\
\hline 30 & 17.06 & --- & --- & 15.61 & --- & 15.71 & 14.87 & --- & 16.27 & 16.25 & 17.01 & 17.34 \\
\hline 31 & 17.02 & --- & --- & 15.55 & --- & 15.72 & --- & --- & --- & 16.29 & 17.04 & --- \\
\hline LOW & --- & --- & --- & --- & 16.05 & 15.91 & 15.79 & --- & --- & 16.49 & 17.04 & 17.34 \\
\hline $\mathrm{HIGH}$ & --- & --- & --- & --- & 15.51 & 15.60 & 14.64 & --- & --- & 15.99 & 16.31 & 17.07 \\
\hline
\end{tabular}


Table 2. Water-level data for monitoring well CW 1983, water years 1996-2001-Continued

[USGS identifier - 435039070261101; ---, no data; m, manual measurement]

WATER YEAR 1997 (OCTOBER 1996 TO SEPTEMBER 1997) DEPTH BELOW LAND SURFACE (WATER LEVEL) (FEET), DAILY MEAN VALUES

\begin{tabular}{|c|c|c|c|c|c|c|c|c|c|c|c|c|}
\hline DAY & $\mathrm{OCT}$ & NOV & $\mathrm{DEC}$ & JAN & FEB & MAR & APR & MAY & JUN & JUL & AUG & SEP \\
\hline 1 & 17.35 & 14.66 & 15.76 & 15.08 & 15.90 & 16.15 & 15.76 & 14.77 & 15.66 & 16.41 & 17.03 & 17.43 \\
\hline 2 & 17.35 & 14.71 & 15.65 & 15.09 & 15.94 & 16.10 & 15.73 & 14.82 & 15.69 & 16.44 & 17.05 & 17.43 \\
\hline 3 & 17.36 & 14.77 & 15.53 & 15.13 & 15.96 & 16.06 & 15.69 & 14.86 & 15.72 & 16.46 & 17.07 & 17.43 \\
\hline 4 & 17.38 & 14.83 & 15.49 & 15.18 & 15.99 & 16.03 & 15.65 & 14.87 & 15.74 & 16.48 & 17.09 & 17.43 \\
\hline 5 & 17.39 & 14.88 & 15.45 & 15.20 & 16.00 & 16.03 & 15.60 & 14.92 & 15.77 & 16.50 & 17.11 & 17.44 \\
\hline 6 & 17.40 & 14.93 & 15.42 & 15.22 & 16.03 & 16.00 & --- & 14.95 & 15.80 & 16.53 & 17.13 & 17.44 \\
\hline 7 & 17.42 & 14.97 & 15.42 & 15.24 & 16.05 & 16.00 & 15.51 & 14.97 & 15.83 & 16.55 & 17.15 & 17.45 \\
\hline 9 & 17.42 & 15.05 & 15.34 & 15.30 & 16.10 & 16.03 & 15.44 & 15.03 & 15.89 & 16.60 & 17.19 & 17.42 \\
\hline 10 & 17.39 & 15.08 & 15.33 & 15.30 & 16.12 & 16.02 & 15.43 & 15.06 & 15.92 & 16.61 & 17.21 & 17.41 \\
\hline 11 & 17.38 & 15.11 & 15.32 & 15.35 & 16.14 & 16.04 & 15.42 & 15.09 & 15.96 & 16.62 & 17.23 & 17.41 \\
\hline 12 & 17.37 & 15.15 & 15.32 & 15.39 & 16.16 & 16.07 & 15.42 & 15.13 & 15.99 & 16.63 & 17.25 & 17.39 \\
\hline 13 & 17.37 & 15.19 & 15.31 & 15.43 & 16.19 & 16.10 & 15.40 & 15.16 & 16.02 & 16.65 & 17.27 & 17.37 \\
\hline 14 & 17.37 & 15.23 & 15.31 & 15.48 & 16.21 & 16.12 & 15.39 & 15.19 & 16.05 & 16.67 & 17.27 & 17.30 \\
\hline 15 & 17.38 & 15.27 & 15.32 & 15.52 & 16.23 & 16.13 & 15.39 & 15.22 & 16.08 & 16.68 & 17.29 & 17.26 \\
\hline 17 & 17.40 & 15.35 & 15.29 & 15.53 & 16.28 & 16.17 & 15.38 & 15.26 & 16.14 & 16.70 & 17.31 & 17.20 \\
\hline 18 & 17.42 & 15.38 & 15.22 & 15.56 & 16.29 & 16.19 & 15.36 & 15.28 & 16.16 & 16.71 & 17.33 & 17.19 \\
\hline 19 & 17.42 & 15.41 & 15.18 & 15.59 & 16.30 & 16.21 & 15.18 & 15.31 & 16.17 & 16.73 & 17.35 & 17.18 \\
\hline 20 & 17.43 & 15.44 & 15.13 & 15.62 & 16.31 & 16.21 & 15.03 & 15.32 & 16.17 & 16.75 & 17.36 & 17.18 \\
\hline 21 & 17.14 & 15.47 & 15.11 & 15.66 & 16.30 & 16.23 & 14.93 & 15.34 & 16.18 & 16.77 & 17.38 & 17.18 \\
\hline 22 & 15.49 & 15.51 & 15.09 & 15.69 & 16.27 & 16.24 & 14.83 & 15.36 & 16.19 & 16.79 & 17.39 & 17.20 \\
\hline 23 & 14.89 & 15.55 & 15.07 & 15.71 & 16.23 & 16.27 & 14.76 & 15.39 & 16.22 & 16.81 & 17.39 & 17.21 \\
\hline 24 & 14.66 & 15.59 & 15.05 & 15.74 & 16.22 & 16.29 & 14.72 & 15.42 & 16.24 & 16.84 & 17.39 & 17.22 \\
\hline 25 & 14.55 & 15.63 & 15.03 & 15.74 & 16.24 & 16.31 & 14.71 & 15.45 & 16.26 & 16.85 & 17.41 & 17.23 \\
\hline 26 & 14.49 & 15.65 & 15.01 & 15.77 & 16.27 & 16.27 & 14.71 & 15.48 & 16.28 & 16.87 & 17.41 & 17.25 \\
\hline 27 & 14.47 & 15.68 & 15.01 & 15.80 & 16.26 & 16.23 & 14.73 & 15.52 & 16.30 & 16.90 & 17.42 & 17.27 \\
\hline 28 & 14.47 & 15.70 & 15.01 & 15.81 & 16.19 & 16.22 & 14.73 & 15.55 & 16.33 & 16.92 & 17.43 & 17.29 \\
\hline 29 & 14.52 & 15.73 & 15.01 & 15.84 & -- & 16.15 & 14.72 & 15.58 & 16.36 & 16.95 & 17.42 & 17.30 \\
\hline 30 & 14.56 & 15.76 & 15.03 & 15.87 & --- & 16.00 & 14.75 & 15.61 & 16.38 & 16.98 & 17.42 & 17.27 \\
\hline 31 & 14.60 & --- & 15.05 & 15.88 & --- & 15.82 & --- & 15.64 & --- & 17.00 & 17.43 & --- \\
\hline
\end{tabular}


Table 2. Water-level data for monitoring well CW 1983, water years 1996-2001-Continued

[USGS identifier - 435039070261101; ---, no data; m, manual measurement]

WATER YEAR 1998 (OCTOBER 1997 TO SEPTEMBER 1998) DEPTH BELOW LAND SURFACE (WATER LEVEL) (FEET), DAILY MEAN VALUES

\begin{tabular}{|c|c|c|c|c|c|c|c|c|c|c|c|c|}
\hline DAY & OCT & NOV & $\mathrm{DEC}$ & JAN & FEB & MAR & APR & MAY & JUN & JUL & AUG & SEP \\
\hline 1 & 17.27 & 17.48 & 16.82 & --- & --- & 15.86 & 15.24 & 15.64 & 16.03 & 14.99 & 16.05 & --- \\
\hline 2 & 17.28 & 17.34 & 16.83 & --- & --- & 15.80 & 15.24 & 15.66 & 16.06 & 15.03 & 16.08 & --- \\
\hline 3 & 17.29 & 17.22 & 16.84 & --- & --- & 15.73 & 15.25 & 15.68 & 16.07 & 15.06 & 16.11 & --- \\
\hline 4 & 17.30 & 17.15 & 16.85 & --- & --- & 15.69 & 15.27 & 15.69 & 16.10 & 15.09 & -- & --- \\
\hline 5 & 17.30 & 17.09 & 16.86 & --- & --- & 15.64 & --- & 15.70 & 16.13 & 15.12 & 16.18 & --- \\
\hline 6 & 17.29 & 17.03 & 16.86 & --- & --- & 15.61 & 15.30 & 15.69 & 16.16 & 15.15 & 16.22 & --- \\
\hline 7 & 17.29 & 16.99 & 16.87 & --- & --- & 15.58 & 15.34 & 15.63 & 16.19 & 15.18 & 16.25 & --- \\
\hline 8 & 17.31 & 16.95 & 16.88 & --- & --- & 15.56 & 15.35 & 15.57 & 16.21 & 15.21 & 16.29 & --- \\
\hline 9 & 17.31 & 16.92 & 16.89 & --- & --- & 15.47 & 15.36 & 15.56 & 16.23 & 15.23 & 16.32 & --- \\
\hline 10 & 17.32 & 16.89 & 16.90 & --- & --- & 15.25 & 15.39 & 15.55 & 16.25 & 15.27 & 16.35 & --- \\
\hline 11 & 17.32 & 16.86 & 16.91 & --- & --- & 15.11 & 15.44 & 15.55 & 16.28 & 15.31 & 16.38 & --- \\
\hline 12 & 17.34 & 16.84 & 16.93 & --- & --- & 15.01 & 15.47 & 15.56 & 16.30 & 15.35 & 16.40 & --- \\
\hline 13 & 17.35 & 16.82 & 16.94 & --- & --- & 14.92 & 15.48 & 15.56 & 16.28 & 15.40 & 16.39 & - \\
\hline 14 & 17.36 & 16.81 & 16.95 & --- & 16.75 & 14.85 & 15.51 & 15.57 & 15.93 & 15.43 & 16.40 & - \\
\hline 15 & 17.37 & 16.80 & 16.97 & --- & 16.73 & 14.84 & 15.54 & 15.58 & 15.57 & 15.48 & 16.41 & --- \\
\hline 16 & 17.38 & 16.79 & 16.98 & --- & 16.72 & 14.85 & 15.57 & 15.62 & 15.38 & 15.52 & 16.42 & --- \\
\hline 17 & 17.39 & 16.79 & 16.99 & --- & 16.70 & 14.86 & 15.58 & 15.64 & 15.21 & 15.55 & 16.44 & - \\
\hline 18 & 17.39 & 16.78 & 17.00 & --- & 16.69 & 14.88 & 15.61 & 15.65 & 15.09 & 15.60 & 16.45 & - \\
\hline 19 & 17.40 & 16.78 & 17.02 & --- & 16.61 & 14.91 & 15.63 & 15.68 & 15.00 & 15.64 & 16.47 & - \\
\hline 20 & 17.41 & 16.79 & 17.03 & --- & 16.54 & 14.94 & 15.60 & 15.71 & 14.93 & 15.67 & 16.49 & --- \\
\hline 21 & 17.42 & 16.80 & 17.05 & --- & 16.49 & 14.98 & 15.59 & 15.72 & 14.90 & 15.71 & 16.51 & --- \\
\hline 22 & 17.43 & 16.80 & 17.06 & --- & 16.48 & 15.01 & 15.59 & 15.76 & 14.88 & 15.75 & 16.53 & -- \\
\hline 23 & 17.44 & 16.80 & 17.07 & --- & 16.44 & 15.08 & 15.59 & 15.78 & 14.87 & 15.79 & 16.56 & - \\
\hline 24 & 17.44 & 16.80 & 17.08 & --- & 16.39 & 15.14 & 15.57 & 15.81 & 14.89 & 15.80 & --- & - \\
\hline 25 & 17.45 & 16.81 & 17.09 & --- & 16.31 & 15.16 & 15.57 & 15.84 & 14.90 & 15.83 & --- & --- \\
\hline 26 & 17.46 & 16.81 & 17.08 & --- & 16.17 & 15.17 & 15.58 & 15.87 & 14.91 & 15.85 & --- & \\
\hline 27 & 17.46 & 16.81 & 17.07 & --- & 16.07 & 15.16 & 15.60 & 15.90 & 14.92 & 15.88 & --- & --- \\
\hline 28 & 17.46 & 16.81 & 17.07 & --- & 15.97 & 15.15 & 15.61 & 15.93 & 14.93 & 15.91 & --- & - \\
\hline 29 & 17.47 & 16.81 & --- & --- & --- & 15.17 & 15.62 & 15.95 & 14.95 & 15.93 & --- & - \\
\hline 30 & 17.47 & 16.82 & --- & --- & --- & 15.19 & 15.63 & 15.99 & 14.96 & 15.98 & --- & - \\
\hline 31 & 17.47 & --- & --- & --- & --- & 15.22 & --- & 16.02 & --- & 16.01 & --- & -- \\
\hline LOW & 17.47 & 17.48 & --- & --- & --- & 15.86 & --- & 16.02 & 16.30 & 16.01 & --- & --- \\
\hline $\mathrm{HIGH}$ & 17.27 & 16.78 & --- & --- & --- & 14.84 & --- & 15.55 & 14.87 & 14.99 & --- & --- \\
\hline
\end{tabular}


Table 2. Water-level data for monitoring well CW 1983, water years 1996-2001-Continued

[USGS identifier - 435039070261101; ---, no data; m, manual measurement]

WATER YEAR 1999 (OCTOBER 1998 TO SEPTEMBER 1999) DEPTH BELOW LAND SURFACE (WATER LEVEL) (FEET), DAILY MEAN VALUES

\begin{tabular}{|c|c|c|c|c|c|c|c|c|c|c|c|c|}
\hline DAY & OCT & NOV & $\mathrm{DEC}$ & JAN & $\mathrm{FEB}$ & MAR & APR & MAY & JUN & JUL & AUG & SEP \\
\hline 1 & 17.01 & 16.23 & 16.42 & 16.74 & 16.19 & 15.95 & 14.88 & --- & --- & --- & --- & 17.62 \\
\hline 2 & 17.03 & 16.25 & 16.42 & 16.76 & 16.20 & 15.76 & 14.88 & --- & --- & --- & --- & 17.63 \\
\hline 3 & 17.04 & 16.27 & 16.42 & 16.76 & 16.12 & 15.69 & 14.87 & --- & --- & --- & --- & 17.64 \\
\hline 4 & 17.06 & 16.29 & 16.43 & 16.77 & 16.02 & 15.56 & m14.88 & --- & --- & --- & --- & 17.66 \\
\hline 5 & 17.07 & 16.32 & 16.45 & 16.79 & 15.98 & 15.38 & 14.92 & --- & --- & --- & --- & 17.67 \\
\hline 6 & 17.09 & 16.34 & 16.46 & 16.80 & 15.94 & 15.29 & 14.95 & --- & --- & --- & --- & 17.68 \\
\hline 7 & 17.10 & 16.36 & 16.48 & 16.81 & 15.91 & 15.22 & 14.97 & --- & --- & --- & --- & 17.69 \\
\hline 9 & 17.12 & 16.41 & 16.52 & 16.83 & 15.90 & 15.15 & 15.03 & --- & --- & --- & --- & 17.71 \\
\hline 10 & 17.03 & 16.43 & 16.53 & 16.81 & 15.90 & 15.14 & 15.07 & --- & --- & --- & m17. 54 & 17.72 \\
\hline 11 & 16.71 & 16.43 & 16.54 & 16.80 & 15.92 & 15.14 & 15.11 & --- & --- & --- & 17.54 & 17.54 \\
\hline 12 & 16.54 & 16.43 & 16.56 & 16.80 & 15.92 & 15.17 & 15.14 & --- & --- & --- & 17.55 & 17.42 \\
\hline 13 & 16.43 & 16.44 & 16.57 & 16.82 & 15.89 & 15.21 & 15.18 & --- & --- & --- & 17.56 & 17.35 \\
\hline 14 & 16.36 & 16.45 & 16.58 & 16.83 & 15.88 & 15.22 & 15.22 & --- & --- & --- & 17.57 & 17.29 \\
\hline 15 & 16.29 & 16.45 & 16.59 & 16.81 & 15.90 & 15.22 & 15.26 & --- & --- & --- & 17.55 & 17.25 \\
\hline 17 & 16.15 & 16.48 & 16.61 & 16.67 & 15.95 & 15.28 & 15.33 & --- & --- & --- & 17.52 & 16.91 \\
\hline 18 & 16.10 & 16.49 & 16.63 & 16.65 & 15.96 & 15.27 & 15.37 & --- & --- & --- & 17.52 & 16.58 \\
\hline 19 & 16.07 & 16.51 & 16.65 & 16.53 & 15.95 & 15.29 & 15.42 & --- & --- & --- & 17.52 & 16.41 \\
\hline 20 & 16.05 & 16.51 & 16.66 & 16.43 & 15.95 & 15.30 & 15.45 & --- & --- & --- & 17.53 & 16.29 \\
\hline 21 & 16.04 & 16.52 & 16.67 & 16.38 & 15.98 & 15.32 & 15.49 & --- & --- & --- & 17.55 & 16.22 \\
\hline 22 & 16.04 & 16.53 & 16.67 & 16.36 & 16.01 & 15.24 & 15.54 & --- & --- & --- & 17.55 & 16.16 \\
\hline 23 & 16.05 & 16.53 & 16.67 & 16.33 & 16.04 & 15.13 & 15.57 & --- & --- & --- & 17.55 & 16.12 \\
\hline 24 & 16.06 & 16.54 & 16.66 & 16.31 & 16.07 & 15.09 & 15.61 & --- & --- & --- & 17.56 & 16.11 \\
\hline 25 & 16.08 & 16.56 & 16.67 & 16.26 & 16.08 & 15.07 & 15.64 & --- & --- & --- & 17.57 & 16.11 \\
\hline 26 & 16.12 & 16.56 & 16.68 & 16.22 & 16.10 & 15.06 & 15.67 & --- & --- & --- & 17.57 & 16.12 \\
\hline 27 & 16.14 & 16.49 & 16.69 & 16.20 & 16.13 & 15.06 & 15.71 & --- & --- & --- & 17.58 & 16.12 \\
\hline 28 & 16.15 & 16.45 & 16.70 & 16.18 & 16.15 & 15.05 & 15.75 & --- & --- & --- & 17.58 & 16.14 \\
\hline 29 & 16.16 & 16.44 & 16.71 & 16.18 & --- & 14.97 & $\operatorname{m15} .78$ & --- & --- & --- & 17.59 & 16.15 \\
\hline 30 & 16.18 & 16.43 & 16.71 & 16.18 & --- & 14.93 & --- & --- & --- & --- & 17.60 & 16.16 \\
\hline 31 & 16.21 & --- & 16.73 & 16.19 & --- & 14.90 & --- & --- & --- & --- & 17.61 & --- \\
\hline
\end{tabular}


Table 2. Water-level data for monitoring well CW 1983, water years 1996-2001-Continued

[USGS identifier - 435039070261101; ---, no data; m, manual measurement]

\section{WATER YEAR 2000 (OCTOBER 1999 TO SEPTEMBER 2000) DEPTH BELOW LAND SURFACE (WATER LEVEL) (FEET), DAILY MEAN VALUES}

\begin{tabular}{|c|c|c|c|c|c|c|c|c|c|c|c|c|}
\hline DAY & $\mathrm{OCT}$ & NOV & $\mathrm{DEC}$ & JAN & $\mathrm{FEB}$ & MAR & APR & MAY & JUN & JUL & AUG & SEP \\
\hline 1 & 16.18 & 16.20 & 16.47 & 16.66 & 16.73 & 16.65 & 15.54 & 15.16 & 15.77 & 16.44 & 16.78 & 17.15 \\
\hline 2 & 16.21 & 16.21 & 16.47 & 16.67 & 16.74 & 16.61 & 15.50 & 15.18 & 15.80 & 16.46 & 16.78 & 17.17 \\
\hline 3 & 16.23 & 16.20 & 16.48 & 16.69 & 16.75 & 16.57 & 15.46 & 15.24 & 15.82 & 16.48 & 16.79 & 17.20 \\
\hline 4 & 16.24 & 16.21 & 16.49 & 16.69 & 16.76 & 16.54 & 15.41 & 15.26 & 15.86 & 16.50 & 16.80 & 17.21 \\
\hline 5 & 16.25 & 16.21 & 16.50 & 16.70 & 16.77 & 16.51 & 15.35 & 15.29 & 15.89 & 16.51 & 16.81 & 17.22 \\
\hline 6 & 16.26 & 16.21 & 16.51 & 16.71 & 16.79 & 16.48 & 15.32 & 15.33 & 15.91 & 16.54 & 16.83 & 17.24 \\
\hline 7 & 16.28 & 16.23 & 16.52 & 16.70 & 16.80 & 16.45 & 15.31 & 15.35 & 15.92 & 16.55 & 16.85 & 17.25 \\
\hline 8 & 16.31 & 16.25 & 16.53 & 16.71 & 16.82 & 16.43 & 15.29 & 15.39 & 15.94 & 16.58 & 16.85 & 17.26 \\
\hline 9 & 16.32 & 16.26 & 16.53 & 16.72 & 16.82 & 16.41 & 15.27 & 15.41 & 15.95 & 16.60 & 16.87 & 17.28 \\
\hline 10 & 16.34 & 16.27 & 16.52 & 16.72 & 16.84 & 16.37 & 15.28 & 15.42 & 15.97 & 16.61 & 16.88 & 17.30 \\
\hline 11 & 16.36 & 16.30 & 16.52 & 16.61 & 16.85 & 16.34 & 15.29 & 15.41 & 16.00 & 16.63 & 16.90 & 17.32 \\
\hline 12 & 16.39 & 16.30 & 16.54 & 16.55 & 16.87 & 16.30 & 15.28 & 15.42 & 16.02 & 16.65 & 16.92 & 17.33 \\
\hline 13 & 16.40 & 16.31 & 16.54 & 16.52 & 16.87 & 16.27 & 15.30 & 15.43 & 16.04 & 16.68 & 16.93 & 17.35 \\
\hline 14 & 16.42 & 16.30 & 16.55 & 16.51 & 16.87 & 16.24 & 15.31 & 15.45 & 16.07 & 16.70 & 16.95 & 17.36 \\
\hline 15 & 16.44 & 16.30 & 16.56 & 16.51 & 16.87 & 16.21 & 15.33 & 15.47 & 16.09 & 16.72 & 16.95 & 17.37 \\
\hline 16 & 16.45 & 16.32 & 16.56 & 16.50 & 16.87 & 16.17 & 15.36 & 15.49 & 16.11 & 16.72 & 16.94 & 17.38 \\
\hline 17 & 16.46 & 16.34 & 16.57 & 16.51 & 16.88 & 16.12 & 15.40 & 15.52 & 16.13 & 16.69 & 16.95 & 17.39 \\
\hline 18 & 16.48 & 16.36 & 16.58 & 16.52 & 16.89 & 16.09 & 15.43 & 15.54 & 16.16 & 16.68 & 16.95 & 17.41 \\
\hline 19 & 16.49 & 16.38 & 16.59 & 16.53 & 16.89 & 16.06 & 15.45 & 15.57 & 16.18 & 16.66 & 16.96 & 17.42 \\
\hline 20 & 16.49 & 16.39 & 16.60 & 16.55 & 16.89 & 16.04 & 15.48 & 15.59 & 16.20 & 16.66 & 16.97 & 17.42 \\
\hline 21 & 16.48 & 16.40 & 16.58 & 16.55 & 16.90 & 16.02 & 15.50 & 15.62 & 16.22 & 16.67 & 16.99 & 17.43 \\
\hline 22 & 16.48 & 16.42 & 16.57 & 16.58 & 16.91 & 16.00 & 15.49 & 15.64 & 16.24 & 16.69 & 17.00 & 17.43 \\
\hline 23 & 16.41 & 16.43 & 16.56 & 16.61 & 16.92 & 15.98 & 15.36 & 15.67 & 16.26 & 16.71 & 17.01 & 17.44 \\
\hline 24 & 16.29 & 16.44 & 16.56 & 16.63 & 16.92 & 15.96 & 15.27 & 15.68 & 16.29 & 16.73 & 17.02 & 17.44 \\
\hline 25 & 16.24 & 16.45 & 16.58 & 16.64 & 16.90 & 15.93 & 15.22 & 15.65 & 16.31 & 16.74 & 17.04 & 17.45 \\
\hline 26 & 16.21 & 16.46 & 16.57 & 16.65 & 16.87 & 15.91 & 15.19 & 15.66 & 16.34 & 16.76 & 17.05 & 17.45 \\
\hline 27 & 16.19 & 16.45 & 16.59 & 16.67 & 16.86 & 15.91 & 15.16 & 15.67 & 16.36 & 16.76 & 17.07 & 17.46 \\
\hline 28 & 16.19 & 16.45 & 16.61 & 16.69 & 16.79 & 15.87 & 15.14 & 15.69 & 16.38 & 16.75 & 17.09 & 17.47 \\
\hline 29 & 16.18 & 16.46 & 16.62 & 16.71 & 16.71 & 15.76 & 15.14 & 15.71 & 16.40 & 16.75 & 17.10 & 17.47 \\
\hline 30 & 16.19 & 16.47 & 16.63 & 16.72 & --- & 15.67 & 15.14 & 15.73 & 16.42 & 16.77 & 17.12 & 17.48 \\
\hline 31 & 16.18 & --- & 16.65 & 16.71 & --- & 15.60 & --- & 15.75 & --- & 16.77 & 17.14 & --- \\
\hline LOW & 16.49 & 16.47 & 16.65 & 16.72 & 16.92 & 16.65 & 15.54 & 15.75 & 16.42 & 16.77 & 17.14 & 17.48 \\
\hline $\mathrm{HIGH}$ & 16.18 & 16.20 & 16.47 & 16.50 & 16.71 & 15.60 & 15.14 & 15.16 & 15.77 & 16.44 & 16.78 & 17.15 \\
\hline
\end{tabular}

WTR YR 2000 HIGH 15.14 LOW 17.48 
Table 2. Water-level data for monitoring well CW 1983, water years 1996-2001-Continued

[USGS identifier - 435039070261101; ---, no data; m, manual measurement]

WATER YEAR 2001 (OCTOBER 2000 TO SEPTEMBER 2001) DEPTH BELOW LAND SURFACE (WATER LEVEL) (FEET), DAILY MEAN VALUES

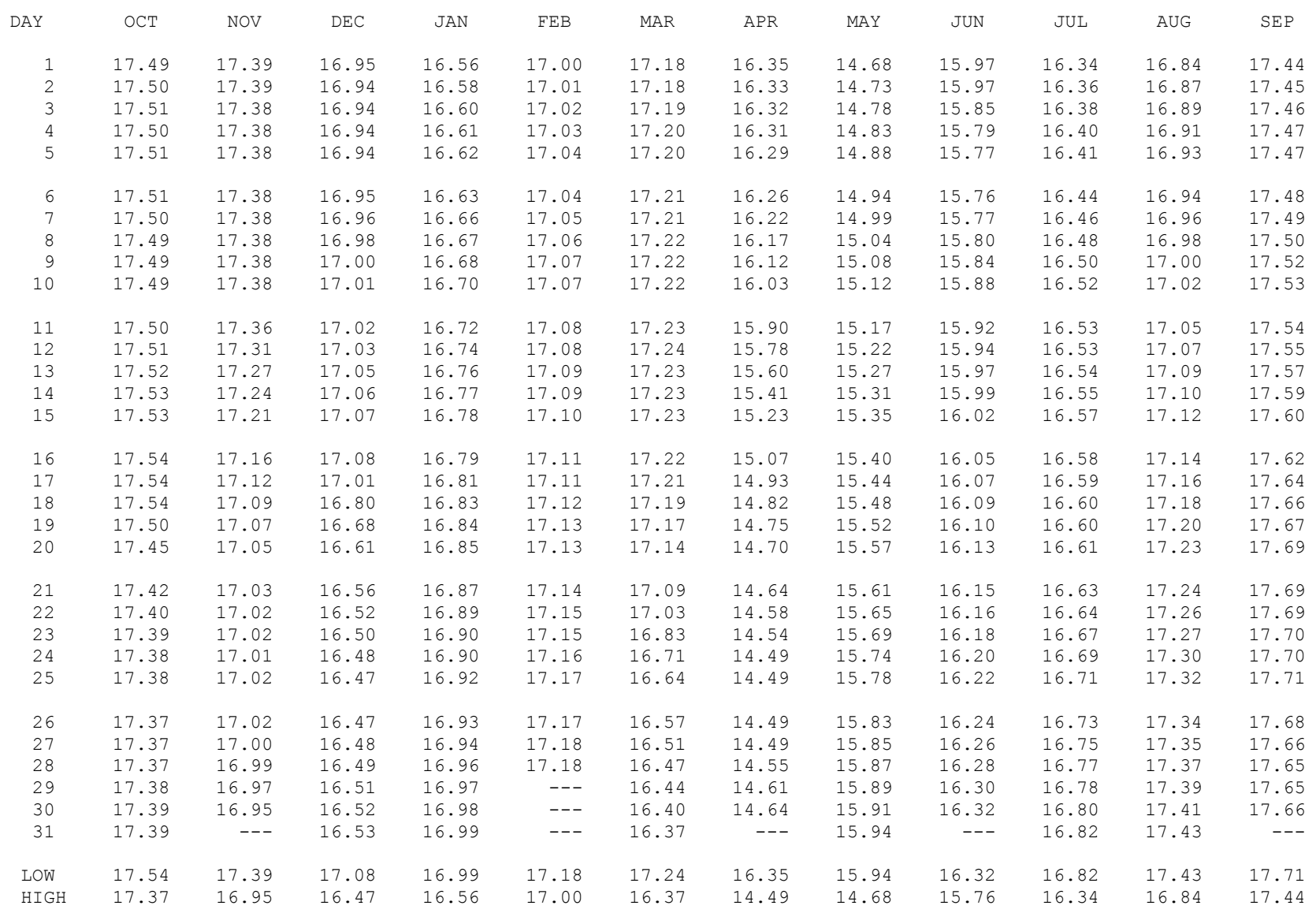

WTR YR 2001 HIGH 14.49 LOW 17.71 
Table 3. Water-quality data from the Windham aquifer, Windham, Maine, 1998-2001 [--, no data available; <, less than; E, estimated value]

\begin{tabular}{|c|c|c|c|c|c|c|c|c|c|c|c|c|}
\hline STATION NUMBER & & $\begin{array}{l}\text { OCAL } \\
\text { ID }\end{array}$ & DATE & TIME & $\begin{array}{l}\text { DEPTH } \\
\text { BELOW } \\
\text { LAND } \\
\text { SURFACE } \\
\text { (WATER } \\
\text { LEVEL) } \\
\text { (FEET) } \\
(72019)\end{array}$ & $\begin{array}{c}\text { BARO- } \\
\text { METRIC } \\
\text { PRES- } \\
\text { SURE } \\
(\text { MM } \\
\text { OF } \\
\text { HG) } \\
(00025)\end{array}$ & $\begin{array}{c}\text { OXYGEN, } \\
\text { DIS- } \\
\text { SOLVED } \\
(M G / L) \\
(00300)\end{array}$ & $\begin{array}{c}\text { PH } \\
\text { WATER } \\
\text { WHOLE } \\
\text { FIELD } \\
\text { (STAND- } \\
\text { ARD } \\
\text { UNITS) } \\
(00400)\end{array}$ & $\begin{array}{l}\text { SPE- } \\
\text { CIFIC } \\
\text { CON- } \\
\text { DUCT- } \\
\text { ANCE } \\
\text { (US/CM) } \\
(00095)\end{array}$ & $\begin{array}{l}\text { TEMPER- } \\
\text { ATURE } \\
\text { WATER } \\
\text { (DEG C) } \\
(00010)\end{array}$ & $\begin{array}{c}\text { NITRO- } \\
\text { GEN, } \\
\text { AMMONIA } \\
\text { DIS- } \\
\text { SOLVED } \\
(\text { MG/L } \\
\text { AS N) } \\
(00608)\end{array}$ & $\begin{array}{l}\text { NITRO- } \\
\text { GEN, AM- } \\
\text { MONIA + } \\
\text { ORGANIC } \\
\text { DIS. } \\
\text { (MG/L } \\
\text { AS N) } \\
(00623)\end{array}$ \\
\hline 435002070255701 & $\mathrm{CW}$ & 1971 & $07-21-98$ & 1630 & 11.71 & 753 & 9.5 & 5.4 & 52 & 18.3 & .040 & $<.10$ \\
\hline 435002070255701 & $\mathrm{CW}$ & 1971 & $09-07-00$ & 1015 & 15.09 & 764 & 5.8 & 5.7 & 37 & 12.7 & $<.020$ & -- \\
\hline 435002070255701 & $\mathrm{CW}$ & 1971 & $08-14-01$ & 1320 & 15.09 & 750 & 5.2 & 5.4 & 19 & 12.6 & $<.040$ & -- \\
\hline 434934070244101 & $\mathrm{CW}$ & 1975 & $07-31-98$ & 1200 & 22.86 & 760 & 8.7 & 8.6 & 108 & 19.8 & .043 & $<.10$ \\
\hline 435008070253901 & CW & 1979 & $08-05-98$ & 1025 & 37.60 & 760 & 3.4 & 6.6 & 263 & 15.3 & .047 & $<.10$ \\
\hline 435008070253901 & $\mathrm{CW}$ & 1979 & $09-06-00$ & 1520 & 38.30 & 769 & 6.5 & 6.7 & 285 & 9.8 & $<.020$ & -- \\
\hline 435008070253901 & $\mathrm{CW}$ & 1979 & $08-21-01$ & 1400 & 38.30 & 749 & 6.2 & 6.3 & 350 & 11.4 & $<.040$ & -- \\
\hline 434919070262601 & $\mathrm{CW}$ & 1980 & $07-30-98$ & 1030 & 14.67 & 752 & 10.5 & 6.0 & 152 & 21.3 & .030 & $<.10$ \\
\hline 434919070262601 & $\mathrm{CW}$ & 1980 & $09-06-00$ & 1010 & 16.99 & 766 & 10.8 & 6.1 & 137 & 12.4 & $<.020$ & -- \\
\hline 434919070262601 & $\mathrm{CW}$ & 1980 & $08-14-01$ & 1100 & 17.48 & 754 & 8.1 & 5.9 & 119 & 14.6 & $<.040$ & -- \\
\hline 435018070250201 & $\mathrm{CW}$ & 1981 & $08-06-98$ & 1320 & 24.77 & 760 & 4.8 & 5.6 & 262 & 15.1 & .043 & $<.10$ \\
\hline 434958070261601 & $\mathrm{CW}$ & 1982 & $08-06-98$ & 1040 & 15.69 & 760 & 7.8 & 7.8 & 169 & 19.2 & .043 & $<.10$ \\
\hline 435039070261101 & $\mathrm{CW}$ & 1983 & $08-04-98$ & 1425 & 16.17 & -- & 11.0 & 6.5 & 90 & 19.6 & .050 & $<.10$ \\
\hline 435039070261101 & $\mathrm{CW}$ & 1983 & $09-12-00$ & 0925 & 17.31 & 756 & 5.9 & 6.4 & 92 & 12.8 & $<.020$ & -- \\
\hline 435039070261101 & $\mathrm{CW}$ & 1983 & $08-16-01$ & 0930 & 17.13 & 757 & 4.5 & 6.2 & 75 & 10.4 & E.035 & -- \\
\hline 435056070263701 & CW & 1984 & $09-02-98$ & 1115 & 4.86 & 752 & 5.6 & 6.5 & 146 & 19.3 & .049 & $<.10$ \\
\hline 435056070263701 & $\mathrm{CW}$ & 1984 & $08-15-01$ & 1025 & 3.89 & 754 & 5.4 & 6.4 & 145 & 9.5 & $<.040$ & -- \\
\hline 435055070263601 & $\mathrm{CW}$ & 1985 & $07-23-98$ & 1035 & 4.14 & 748 & .6 & 5.9 & 66 & 25.8 & .042 & .13 \\
\hline 435055070263601 & $\mathrm{CW}$ & 1985 & $09-05-00$ & 0945 & 3.94 & 764 & .2 & 5.9 & 77 & 10.8 & $<.020$ & -- \\
\hline 435055070263601 & $\mathrm{CW}$ & 1985 & $09-12-00$ & 1140 & 3.96 & 757 & 2.1 & 6.0 & 79 & 14.2 & $<.020$ & -- \\
\hline 435055070263601 & $\mathrm{CW}$ & 1985 & $08-15-01$ & 1200 & 4.04 & 754 & .4 & 5.8 & 60 & 11.4 & $<.040$ & -- \\
\hline 434910070255601 & CW & 1987 & $09-03-98$ & 1020 & 58.35 & 752 & 1.1 & 7.5 & 225 & 13.4 & .036 & $<.10$ \\
\hline 434910070255601 & $\mathrm{CW}$ & 1987 & $09-08-00$ & 1425 & 59.02 & 754 & 2.7 & 7.2 & 197 & 16.3 & $<.020$ & -- \\
\hline 434910070255601 & $\mathrm{CW}$ & 1987 & $08-22-01$ & 1430 & 59.06 & 749 & .4 & 7.6 & 229 & 12.0 & $<.040$ & -- \\
\hline 434941070261901 & $\mathrm{CW}$ & 1988 & $09-01-98$ & 1345 & 2.15 & 754 & 2.9 & 5.5 & 145 & 14.4 & .021 & $<.10$ \\
\hline 434919070262602 & $\mathrm{CW}$ & 1989 & $07-30-98$ & 1255 & 11.49 & 752 & 10.9 & 5.6 & 109 & 20.9 & .037 & $<.10$ \\
\hline 435002070255601 & CW & 1990 & $07-28-98$ & 1405 & 25.72 & 751 & 6.1 & 8.8 & 185 & 23.0 & .045 & $<.10$ \\
\hline 435002070255601 & CW & 1990 & $09-07-00$ & 1125 & 27.41 & 764 & .1 & 7.2 & 287 & 12.9 & $<.020$ & -- \\
\hline 435002070255601 & $\mathrm{CW}$ & 1990 & $08-14-01$ & 1415 & 27.51 & 750 & .4 & 7.0 & 312 & 14.1 & E.030 & -- \\
\hline 435008070262901 & CW & 1992 & $07-22-98$ & 1310 & 10.82 & 748 & 7.3 & 5.8 & 39 & 17.5 & .023 & $<.10$ \\
\hline 435008070262901 & $\mathrm{CW}$ & 1992 & $09-05-00$ & 1255 & 13.27 & 764 & 7.6 & 5.5 & 75 & 10.7 & $<.020$ & -- \\
\hline 435008070262901 & $\mathrm{CW}$ & 1992 & 08-13-01 & 1230 & 13.41 & 752 & 6.1 & 5.4 & 63 & 15.3 & $<.040$ & -- \\
\hline 435008070262902 & $\mathrm{CW}$ & 1993 & $07-22-98$ & 1510 & 8.02 & 748 & 9.0 & 5.8 & 40 & 22.3 & .032 & $<.10$ \\
\hline 435008070262902 & CW & 1993 & $09-05-00$ & 1150 & 10.30 & 764 & 7.4 & 5.5 & 84 & 10.7 & $<.020$ & -- \\
\hline 435008070262902 & $\mathrm{CW}$ & 1993 & $08-13-01$ & 1320 & 10.23 & 752 & 7.3 & 5.3 & 48 & 10.5 & $<.040$ & -- \\
\hline 434945070263401 & $\mathrm{CW}$ & 1994 & $08-31-98$ & 1430 & 1.36 & 754 & 5.8 & 6.3 & 252 & 22.5 & $<.020$ & $<.10$ \\
\hline 435012070265101 & $\mathrm{CW}$ & 1995 & $08-31-98$ & 1120 & 13.26 & 754 & 4.8 & 5.7 & 204 & 14.7 & $<.020$ & $<.10$ \\
\hline 435027070264801 & $\mathrm{CW}$ & 1998 & $07-29-98$ & 1620 & 13.03 & 747 & 9.6 & 5.9 & 43 & 22.6 & $<.020$ & $<.10$ \\
\hline 435026070264101 & CW & 1999 & $07-21-98$ & 1030 & 21.73 & 753 & 1.6 & 7.9 & 136 & 20.7 & .346 & .34 \\
\hline 435026070264101 & CW & 1999 & $09-11-00$ & 0840 & 22.82 & 760 & 1.0 & 8.3 & 112 & 12.9 & $<.020$ & -- \\
\hline 435026070264101 & $\mathrm{CW}$ & 1999 & $08-14-01$ & 0945 & 22.62 & 753 & 1.1 & 8.1 & 102 & 12.8 & E. 026 & -- \\
\hline
\end{tabular}


Table 3. Water-quality data from the Windham aquifer, Windham, Maine, 1998-2001—Continued $[--$, no data available; $<$, less than; E, estimated value]

\begin{tabular}{|c|c|c|c|c|c|c|c|c|c|}
\hline STATION & & DCAL & DATE & TIME & $\begin{array}{c}\text { NITRO- } \\
\text { GEN, } \\
\text { NO2+NO3 } \\
\text { DIS- } \\
\text { SOLVED } \\
\text { (MG/L } \\
\text { AS N) } \\
(00631)\end{array}$ & $\begin{array}{c}\text { NITRO- } \\
\text { GEN, } \\
\text { NITRITE } \\
\text { DIS- } \\
\text { SOLVED } \\
\text { (MG/L } \\
\text { AS N) } \\
(00613)\end{array}$ & $\begin{array}{c}\text { PHOS- } \\
\text { PHORUS } \\
\text { DIS- } \\
\text { SOLVED } \\
(\text { MG/L } \\
\text { AS P) } \\
(00666)\end{array}$ & $\begin{array}{c}\text { PHOS- } \\
\text { PHORUS } \\
\text { ORTHO, } \\
\text { DIS- } \\
\text { SOLVED } \\
(\text { MG/L } \\
\text { AS P) } \\
(00671)\end{array}$ & $\begin{array}{c}\text { ARSENIC } \\
\text { DIS- } \\
\text { SOLVED } \\
(\text { UG } / L \\
\text { AS AS) } \\
(01000)\end{array}$ \\
\hline 435002070255701 & $\mathrm{CW}$ & 1971 & $07-21-98$ & 1630 & .288 & $<.010$ & -- & -- & -- \\
\hline 435002070255701 & $\mathrm{CW}$ & 1971 & $09-07-00$ & 1015 & .108 & $<.010$ & .018 & .014 & $<.9$ \\
\hline 435002070255701 & $\mathrm{CW}$ & 1971 & $08-14-01$ & 1320 & .269 & $<.006$ & .012 & $<.020$ & -- \\
\hline 434934070244101 & $\mathrm{CW}$ & 1975 & $07-31-98$ & 1200 & .068 & $<.010$ & -- & -- & -- \\
\hline 435008070253901 & $\mathrm{CW}$ & 1979 & $08-05-98$ & 1025 & 3.06 & $<.010$ & -- & -- & -- \\
\hline 435008070253901 & $\mathrm{CW}$ & 1979 & $09-06-00$ & 1520 & .586 & $<.010$ & E. 004 & $<.010$ & $<.9$ \\
\hline 435008070253901 & $\mathrm{CW}$ & 1979 & $08-21-01$ & 1400 & 2.67 & $<.006$ & $<.006$ & $<.020$ & -- \\
\hline 434919070262601 & $\mathrm{CW}$ & 1980 & $07-30-98$ & 1030 & 6.43 & $<.010$ & -- & -- & -- \\
\hline 434919070262601 & $\mathrm{CW}$ & 1980 & $09-06-00$ & 1010 & 6.25 & $<.010$ & E. 005 & $<.010$ & $<.9$ \\
\hline 434919070262601 & $\mathrm{CW}$ & 1980 & $08-14-01$ & 1100 & 4.84 & $<.006$ & E.004 & $<.020$ & -- \\
\hline 435018070250201 & $\mathrm{CW}$ & 1981 & $08-06-98$ & 1320 & 2.29 & .010 & -- & -- & -- \\
\hline 434958070261601 & $\mathrm{CW}$ & 1982 & $08-06-98$ & 1040 & $<.050$ & $<.010$ & -- & -- & -- \\
\hline 435039070261101 & $\mathrm{CW}$ & 1983 & $08-04-98$ & 1425 & .484 & $<.010$ & -- & -- & -- \\
\hline 435039070261101 & $\mathrm{CW}$ & 1983 & $09-12-00$ & 0925 & .435 & $<.010$ & E. 004 & $<.010$ & $<.9$ \\
\hline 435039070261101 & $\mathrm{CW}$ & 1983 & $08-16-01$ & 0930 & .469 & $<.006$ & $<.006$ & $<.020$ & -- \\
\hline 435056070263701 & $\mathrm{CW}$ & 1984 & $09-02-98$ & 1115 & 1.13 & $<.010$ & -- & -- & -- \\
\hline 435056070263701 & $\mathrm{CW}$ & 1984 & $08-15-01$ & 1025 & 1.18 & $<.006$ & $<.006$ & $<.020$ & -- \\
\hline 435055070263601 & $\mathrm{CW}$ & 1985 & $07-23-98$ & 1035 & $<.050$ & $<.010$ & -- & -- & -- \\
\hline 435055070263601 & $\mathrm{CW}$ & 1985 & $09-05-00$ & 0945 & $<.050$ & $<.010$ & $<.006$ & $<.010$ & $<.9$ \\
\hline 435055070263601 & $\mathrm{CW}$ & 1985 & $09-12-00$ & 1140 & $<.050$ & $<.010$ & $<.006$ & $<.010$ & $<.9$ \\
\hline 435055070263601 & $\mathrm{CW}$ & 1985 & $08-15-01$ & 1200 & $<.050$ & $<.006$ & $<.006$ & $<.020$ & -- \\
\hline 434910070255601 & $\mathrm{CW}$ & 1987 & $09-03-98$ & 1020 & .488 & .012 & -- & -- & -- \\
\hline 434910070255601 & $\mathrm{CW}$ & 1987 & $09-08-00$ & 1425 & $<.050$ & $<.010$ & $<.006$ & $<.010$ & 1.6 \\
\hline 434910070255601 & $\mathrm{CW}$ & 1987 & $08-22-01$ & 1430 & .057 & $<.006$ & $<.006$ & $<.020$ & -- \\
\hline 434941070261901 & $\mathrm{CW}$ & 1988 & $09-01-98$ & 1345 & .859 & $<.010$ & -- & -- & -- \\
\hline 434919070262602 & $\mathrm{CW}$ & 1989 & $07-30-98$ & 1255 & 4.16 & $<.010$ & -- & -- & -- \\
\hline 435002070255601 & $\mathrm{CW}$ & 1990 & $07-28-98$ & 1405 & .055 & $<.010$ & -- & -- & -- \\
\hline 435002070255601 & $\mathrm{CW}$ & 1990 & $09-07-00$ & 1125 & $<.050$ & $<.010$ & E.003 & $<.010$ & 5.0 \\
\hline 435002070255601 & $\mathrm{CW}$ & 1990 & $08-14-01$ & 1415 & E.028 & $<.006$ & $<.006$ & $<.020$ & -- \\
\hline 435008070262901 & $\mathrm{CW}$ & 1992 & $07-22-98$ & 1310 & .398 & $<.010$ & -- & -- & -- \\
\hline 435008070262901 & $\mathrm{CW}$ & 1992 & $09-05-00$ & 1255 & 1.39 & $<.010$ & $<.006$ & $<.010$ & $<.9$ \\
\hline 435008070262901 & $\mathrm{CW}$ & 1992 & $08-13-01$ & 1230 & 1.40 & $<.006$ & $<.006$ & $<.020$ & -- \\
\hline 435008070262902 & $\mathrm{CW}$ & 1993 & $07-22-98$ & 1510 & .685 & $<.010$ & -- & -- & -- \\
\hline 435008070262902 & $\mathrm{CW}$ & 1993 & $09-05-00$ & 1150 & 1.50 & $<.010$ & $<.006$ & $<.010$ & $<.9$ \\
\hline 435008070262902 & $\mathrm{CW}$ & 1993 & $08-13-01$ & 1320 & 1.43 & $<.006$ & $<.006$ & $<.020$ & -- \\
\hline 434945070263401 & $\mathrm{CW}$ & 1994 & $08-31-98$ & 1430 & .994 & $<.010$ & -- & -- & -- \\
\hline 435012070265101 & $\mathrm{CW}$ & 1995 & $08-31-98$ & 1120 & 3.76 & $<.010$ & -- & -- & -- \\
\hline 435027070264801 & $\mathrm{CW}$ & 1998 & $07-29-98$ & 1620 & .426 & $<.010$ & -- & -- & -- \\
\hline 435026070264101 & $\mathrm{CW}$ & 1999 & $07-21-98$ & 1030 & .169 & $<.010$ & -- & -- & -- \\
\hline 435026070264101 & $\mathrm{CW}$ & 1999 & $09-11-00$ & 0840 & .151 & $<.010$ & .046 & .037 & 2.8 \\
\hline 435026070264101 & $\mathrm{CW}$ & 1999 & $08-14-01$ & 0945 & .156 & $<.006$ & .041 & .034 & -- \\
\hline
\end{tabular}


Table 3. Water-quality data from the Windham aquifer, Windham, Maine, 1998-2001—Continued [--, no data available; $<$, less than; E, estimated value]

\begin{tabular}{|c|c|c|c|c|c|c|c|c|c|c|c|c|}
\hline STATION NUMBER & & $\begin{array}{l}\text { DCAL } \\
\text { ID }\end{array}$ & DATE & TIME & $\begin{array}{l}\text { DEPTH } \\
\text { BELOW } \\
\text { LAND } \\
\text { SUREACE } \\
\text { (WATER } \\
\text { LEVEL) } \\
\text { (FEET) } \\
(72019)\end{array}$ & $\begin{array}{c}\text { BARO- } \\
\text { METRIC } \\
\text { PRES- } \\
\text { SURE } \\
(\text { MM } \\
\text { OF } \\
\text { HG) } \\
(00025)\end{array}$ & $\begin{array}{c}\text { OXYGEN, } \\
\text { DIS- } \\
\text { SOLVED } \\
(\mathrm{MG} / \mathrm{L}) \\
(00300)\end{array}$ & $\begin{array}{c}\text { PH } \\
\text { WATER } \\
\text { WHOLE } \\
\text { FIELD } \\
\text { (STAND- } \\
\text { ARD } \\
\text { UNITS) } \\
(00400)\end{array}$ & $\begin{array}{l}\text { SPE- } \\
\text { CIFIC } \\
\text { CON- } \\
\text { DUCT- } \\
\text { ANCE } \\
\text { (US/CM) } \\
(00095)\end{array}$ & $\begin{array}{c}\text { TEMPER- } \\
\text { ATURE } \\
\text { WATER } \\
(\text { DEG C) } \\
(00010)\end{array}$ & $\begin{array}{c}\text { NITRO- } \\
\text { GEN, } \\
\text { AMMONIA } \\
\text { DIS- } \\
\text { SOLVED } \\
\text { (MG/L } \\
\text { AS N) } \\
(00608)\end{array}$ & $\begin{array}{l}\text { NITRO- } \\
\text { GEN, AM- } \\
\text { MONIA + } \\
\text { ORGANIC } \\
\text { DIS. } \\
\text { (MG/L } \\
\text { AS N) } \\
(00623)\end{array}$ \\
\hline 435041070262301 & CW & 2000 & $07-22-98$ & 0940 & 26.83 & 748 & 2.1 & 6.1 & 562 & 17.6 & .028 & .23 \\
\hline 435041070262301 & CW & 2000 & $09-06-00$ & 1135 & 27.92 & 769 & .3 & 6.4 & 604 & 11.8 & $<.020$ & -- \\
\hline 435041070262301 & & 2000 & $08-15-01$ & 0840 & 27.76 & 755 & .5 & 6.3 & 566 & 11.9 & $<.040$ & -- \\
\hline 435131070261401 & CW & 2001 & $08-10-98$ & 1155 & 14.54 & 754 & .8 & 8.7 & 309 & 14.9 & .055 & $<.10$ \\
\hline 435131070261401 & CW & 2001 & $09-11-00$ & 1125 & 14.93 & 760 & .2 & 8.8 & 318 & 10.3 & .024 & -- \\
\hline 435131070261401 & $\mathrm{CW}$ & 2001 & $08-15-01$ & 1350 & 15.02 & 755 & .4 & 8.5 & 343 & 12.0 & $<.040$ & -- \\
\hline 434946070252301 & CW & 2003 & $08-10-98$ & 1505 & 44.70 & 752 & 3.6 & 6.4 & 146 & 18.1 & .055 & $<.10$ \\
\hline 434946070252301 & CW & 2003 & $09-07-00$ & 1450 & 45.68 & 765 & 2.8 & 6.6 & 122 & 16.4 & $<.020$ & -- \\
\hline 434946070252301 & CW & 2003 & $08-21-01$ & 1530 & 45.81 & 750 & 3.6 & 6.1 & 105 & 14.3 & $<.040$ & -- \\
\hline 434927070255101 & CW & 2004 & $07-29-98$ & 1055 & 49.14 & 747 & 10.1 & 5.7 & 103 & 18.4 & $<.020$ & $<.10$ \\
\hline 434927070255101 & CW & 2004 & $09-05-00$ & 1410 & 50.51 & 764 & 8.7 & 5.4 & 114 & 14.1 & $<.020$ & -- \\
\hline 434927070255101 & $\mathrm{CW}$ & 2004 & $08-22-01$ & 0900 & 50.83 & 754 & 8.8 & 5.4 & 151 & 16.7 & $<.040$ & -- \\
\hline 435008070253902 & CW & 2005 & $08-05-98$ & 1245 & 34.55 & 760 & 10.3 & 5.7 & 164 & 18.1 & .045 & $<.10$ \\
\hline 435008070253902 & CW & 2005 & $09-06-00$ & 1345 & 36.18 & 765 & 9.3 & 5.6 & 184 & 13.4 & $<.020$ & -- \\
\hline 435008070253902 & CW & 2005 & $08-21-01$ & 1300 & 36.26 & 749 & 9.5 & 5.3 & 257 & 14.5 & $<.040$ & -- \\
\hline 435040070264501 & CW & 2007 & $07-30-98$ & 1540 & 9.39 & 753 & 9.6 & 5.4 & 328 & 17.5 & .037 & $<.10$ \\
\hline 435040070264501 & CW & 2007 & $09-11-00$ & 1415 & 9.97 & 757 & 9.5 & 5.7 & 346 & 14.9 & $<.020$ & -- \\
\hline 435131070261402 & CW & 2008 & $08-10-98$ & 0940 & 13.35 & 754 & 9.8 & 5.6 & 36 & 13.6 & .058 & $<.10$ \\
\hline 435131070261402 & CW & 2008 & $09-11-00$ & 1020 & 13.57 & 756 & 10.5 & 5.4 & 109 & 11.7 & $<.020$ & -- \\
\hline 435131070261402 & CW & 2008 & $08-15-01$ & 1445 & 13.55 & 755 & 6.1 & 5.4 & 13 & 12.3 & $<.040$ & -- \\
\hline 435026070264102 & CW & 2009 & $07-20-98$ & 1430 & 11.40 & 750 & 10.8 & 5.9 & 52 & 15.4 & .036 & $<.10$ \\
\hline 435026070264102 & CW & 2009 & $09-08-00$ & 1040 & 14.25 & 756 & 5.1 & 5.4 & 221 & 14.2 & .822 & -- \\
\hline 435026070264102 & $\mathrm{CW}$ & 2009 & $08-14-01$ & 0850 & 13.81 & 753 & 4.5 & 5.2 & 295 & 13.6 & .223 & -- \\
\hline 435018070263201 & CW & 2010 & $07-28-98$ & 1110 & 14.29 & 750 & 9.8 & 5.7 & 87 & 19.5 & .031 & $<.10$ \\
\hline 435018070263201 & CW & 2010 & $09-07-00$ & 0900 & 16.38 & 764 & 7.5 & 5.7 & 191 & 12.8 & $<.020$ & -- \\
\hline 435018070263201 & CW & 2010 & $08-22-01$ & 1025 & 16.27 & 751 & 7.3 & 5.4 & 220 & 14.6 & $<.040$ & -- \\
\hline 435014070264901 & CW & 2011 & $07-21-98$ & 1400 & 11.58 & 753 & 9.8 & 5.6 & 38 & 17.2 & .041 & $<.10$ \\
\hline 435014070264901 & CW & 2011 & $09-06-00$ & 0900 & 13.99 & 769 & 11.2 & 5.6 & 49 & 11.0 & $<.020$ & -- \\
\hline 435014070264901 & $\mathrm{CW}$ & 2011 & $08-13-01$ & 1415 & 13.84 & 752 & 10.6 & 5.4 & 59 & 15.7 & E. 024 & -- \\
\hline 435004070262101 & CW & 2012 & $09-03-98$ & 1400 & 14.58 & 752 & 8.7 & 6.1 & 465 & 18.8 & .036 & .11 \\
\hline 435004070262101 & CW & 2012 & $08-22-01$ & 1235 & 16.07 & 751 & 1.0 & 6.5 & 487 & 14.6 & $<.040$ & -- \\
\hline 435136070271201 & CW & 2019 & $09-01-98$ & 1010 & 5.54 & 754 & 6.7 & 6.2 & 58 & 14.0 & .021 & $<.10$ \\
\hline
\end{tabular}


Table 3. Water-quality data from the Windham aquifer, Windham, Maine, 1998-2001—Continued $[--$, no data available; < , less than; E, estimated value]

\begin{tabular}{|c|c|c|c|c|c|c|c|c|}
\hline STATION & $\begin{array}{l}\text { LOCAL } \\
\text { ID }\end{array}$ & DATE & TIME & $\begin{array}{c}\text { NITRO- } \\
\text { GEN, } \\
\text { NO2+NO3 } \\
\text { DIS- } \\
\text { SOLVED } \\
(\mathrm{MG} / \mathrm{L} \\
\text { AS N) } \\
(00631)\end{array}$ & $\begin{array}{c}\text { NITRO- } \\
\text { GEN, } \\
\text { NITRITE } \\
\text { DIS- } \\
\text { SOLVED } \\
(\text { MG/L } \\
\text { AS N) } \\
(00613)\end{array}$ & $\begin{array}{c}\text { PHOS- } \\
\text { PHORUS } \\
\text { DIS- } \\
\text { SOLVED } \\
(\text { MG/L } \\
\text { AS P) } \\
(00666)\end{array}$ & $\begin{array}{c}\text { PHOS- } \\
\text { PHORUS } \\
\text { ORTHO, } \\
\text { DIS- } \\
\text { SOLVED } \\
(\text { MG/L } \\
\text { AS P) } \\
(00671)\end{array}$ & $\begin{array}{c}\text { ARSENIC } \\
\text { DIS- } \\
\text { SOLVED } \\
(\text { UG / L } \\
\text { AS AS) } \\
(01000)\end{array}$ \\
\hline 435041070262301 & CW 2000 & $07-22-98$ & 0940 & 9.56 & .136 & -- & -- & -- \\
\hline 435041070262301 & CW 2000 & $09-06-00$ & 1135 & 26.6 & .153 & .008 & $<.010$ & $<.9$ \\
\hline 435041070262301 & CW 2000 & $08-15-01$ & 0840 & 14.0 & .037 & .009 & $<.020$ & -- \\
\hline 435131070261401 & CW 2001 & $08-10-98$ & 1155 & $<.050$ & $<.010$ & -- & -- & -- \\
\hline 435131070261401 & CW 2001 & $09-11-00$ & 1125 & $<.050$ & $<.010$ & .337 & .323 & 3.6 \\
\hline 435131070261401 & CW 2001 & $08-15-01$ & 1350 & E. 024 & E.003 & .440 & .435 & -- \\
\hline 434946070252301 & CW 2003 & $08-10-98$ & 1505 & 1.97 & $<.010$ & -- & -- & -- \\
\hline 434946070252301 & CW 2003 & $09-07-00$ & 1450 & .964 & $<.010$ & $<.006$ & $<.010$ & .9 \\
\hline 434946070252301 & CW 2003 & $08-21-01$ & 1530 & 1.43 & $<.006$ & $<.006$ & $<.020$ & -- \\
\hline 434927070255101 & CW 2004 & $07-29-98$ & 1055 & 2.77 & $<.010$ & -- & -- & -- \\
\hline 434927070255101 & CW 2004 & $09-05-00$ & 1410 & 3.71 & $<.010$ & .008 & $<.010$ & $<.9$ \\
\hline 434927070255101 & CW 2004 & $08-22-01$ & 0900 & 3.71 & $<.006$ & E.006 & E.010 & -- \\
\hline 435008070253902 & CW 2005 & $08-05-98$ & 1245 & 4.39 & $<.010$ & -- & -- & -- \\
\hline 435008070253902 & CW 2005 & $09-06-00$ & 1345 & 1.24 & $<.010$ & .006 & $<.010$ & $<.9$ \\
\hline 435008070253902 & CW 2005 & $08-21-01$ & 1300 & 3.96 & $<.006$ & E.004 & $<.020$ & -- \\
\hline 435040070264501 & CW 2007 & $07-30-98$ & 1540 & 1.35 & $<.010$ & -- & -- & -- \\
\hline 435040070264501 & CW 2007 & $09-11-00$ & 1415 & .601 & $<.010$ & E.003 & $<.010$ & $<.9$ \\
\hline 435131070261402 & CW 2008 & $08-10-98$ & 0940 & .332 & $<.010$ & -- & -- & -- \\
\hline 435131070261402 & CW 2008 & $09-11-00$ & 1020 & 1.82 & $<.010$ & $<.006$ & $<.010$ & $<.9$ \\
\hline 435131070261402 & CW 2008 & $08-15-01$ & 1445 & .505 & $<.006$ & E.003 & $<.020$ & -- \\
\hline 435026070264102 & CW 2009 & $07-20-98$ & 1430 & 1.67 & $<.010$ & -- & -- & -- \\
\hline 435026070264102 & CW 2009 & $09-08-00$ & 1040 & 13.5 & $<.010$ & $<.006$ & $<.010$ & $<.9$ \\
\hline 435026070264102 & CW 2009 & $08-14-01$ & 0850 & 13.6 & .015 & .011 & $<.020$ & -- \\
\hline 435018070263201 & CW 2010 & $07-28-98$ & 1110 & .848 & $<.010$ & -- & -- & -- \\
\hline 435018070263201 & CW 2010 & $09-07-00$ & 0900 & .932 & $<.010$ & E.003 & $<.010$ & $<.9$ \\
\hline 435018070263201 & CW 2010 & $08-22-01$ & 1025 & 1.27 & $<.006$ & $<.006$ & $<.020$ & -- \\
\hline 435014070264901 & CW 2011 & $07-21-98$ & 1400 & .280 & $<.010$ & -- & -- & -- \\
\hline 435014070264901 & CW 2011 & $09-06-00$ & 0900 & .134 & $<.010$ & $<.006$ & $<.010$ & $<.9$ \\
\hline 435014070264901 & CW 2011 & $08-13-01$ & 1415 & .410 & $<.006$ & $<.006$ & $<.020$ & -- \\
\hline 435004070262101 & CW 2012 & $09-03-98$ & 1400 & 4.33 & .014 & -- & -- & -- \\
\hline 435004070262101 & CW 2012 & $08-22-01$ & 1235 & 4.57 & $<.006$ & E.004 & $<.020$ & -- \\
\hline 435136070271201 & CW 2019 & $09-01-98$ & 1010 & $<.050$ & $<.010$ & -- & -- & -- \\
\hline
\end{tabular}



District Chief

Maine District

U.S. Geological Survey

Water Resources Division

26 Ganneston Drive

Augusta, Maine 04330

8 Printed on recycled paper 